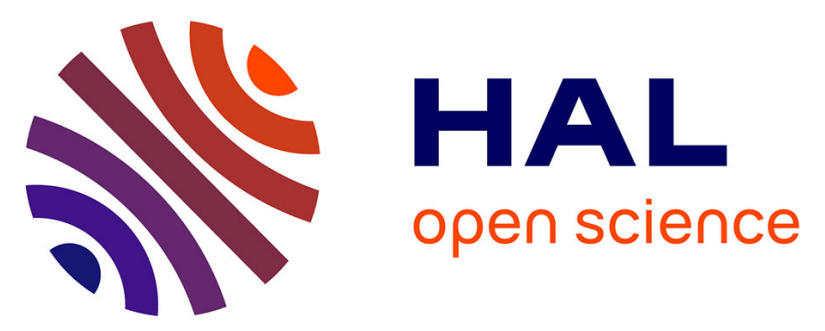

\title{
Magnetic anisotropy, exchange and damping in cobalt-based full-Heusler compounds: an experimental review
}

Simon Trudel, Oksana Gaier, Jaroslav Hamrle, Burkard Hillebrands

\section{- To cite this version:}

Simon Trudel, Oksana Gaier, Jaroslav Hamrle, Burkard Hillebrands. Magnetic anisotropy, exchange and damping in cobalt-based full-Heusler compounds: an experimental review. Journal of Physics D: Applied Physics, 2010, 43 (19), pp.193001. 10.1088/0022-3727/43/19/193001 . hal-00569597

\section{HAL Id: hal-00569597 \\ https://hal.science/hal-00569597}

Submitted on 25 Feb 2011

HAL is a multi-disciplinary open access archive for the deposit and dissemination of scientific research documents, whether they are published or not. The documents may come from teaching and research institutions in France or abroad, or from public or private research centers.
L'archive ouverte pluridisciplinaire HAL, est destinée au dépôt et à la diffusion de documents scientifiques de niveau recherche, publiés ou non, émanant des établissements d'enseignement et de recherche français ou étrangers, des laboratoires publics ou privés. 


\title{
Magnetic anisotropy, exchange and damping in cobalt-based full Heusler compounds: An experimental review
}

\author{
Simon Trudel, ${ }^{1,2}$ Oksana Gaier, ${ }^{2}$ Jaroslav Hamrle, ${ }^{2}$ and Burkard Hillebrands ${ }^{2}$ \\ 1 Department of Chemistry, University of Calgary, \\ 2500 University Dr. NW, Calgary AB Canada T2N 1N4, \\ 2 Department of Physics and Research Center OPTIMAS, \\ Technische Universität Kaiserslautern, 67663 Kaiserslautern, Germany*
}

\begin{abstract}
Cobalt-based full-Heusler compounds with composition $\mathrm{Co}_{2} M^{\prime} Z$ (where $M^{\prime}$ is a transition metal and $Z$ is a main group element) are attracting attention due to their predicted half-metallic behaviour, a considerably desired property for spin-dependent electron transport devices. Knowledge of the basic magnetic properties of these materials, in particular in the form of thin films, is required both to fully exploit these promising materials, and to understand their underlying electronic structure and establish structure-property relationships. In this Topical Review, we present a survey of the magnetic anisotropy, exchange, and damping of $\mathrm{Co}_{2} M^{\prime} Z$ compounds. These properties are directly related to spin-spin and spin-orbit interactions.
\end{abstract}

\section{INTRODUCTION}

The interest in the intermetallic compounds with the general formula $M_{2} M^{\prime} Z$, where $M$ and $M^{\prime}$ are transition metals and $Z$ is a main-group element, ignited in 1903 when Friedrich Heusler discovered that $\mathrm{Cu}_{2} \mathrm{MnAl}$ was ferromagnetic, even though it does not contain any of the pure ferromagnetic elements [1]. Since this discovery, numerous investigations were made to clarify the chemical and magnetic ordering in these materials [2-5], and different models were proposed to shed light on the microscopic interactions leading to the appearance of ferromagnetism [6-8]. It was also found that some ternary intermetallic compounds with the chemical composition $M M^{\prime} Z$ exhibit ferromagnetic behaviour as well. Today this latter class of materials is known as half-Heusler compounds, whereas the $M_{2} M^{\prime} Z$ compounds are named full-Heusler compounds. Amongst the half-Heusler compounds, PtMnSb attracted particular attention due to its extremely large magneto-optical Kerr rotation [9], which made this compound a suitable material for magnetooptical recording technology.

In 1983, de Groot et al. [11] calculated the band structure of NiMnSb, and termed this material to be a "half metal" (not to be confused with a semi-metal such as bismuth). A general introduction to half-metallic ferromagnets is offered by Coey et al. [12]. Here we refer to Fig. 1 [10] which shows the calculated band structure of $\mathrm{Co}_{2} \mathrm{FeSi}$, a more contemporary example closer to the topic of this Review, to illustrate what a half-metal is. First, one considers the spin-resolved band structures. The majority spin band (Fig. 1c) exhibits metallic character, i.e. the valence band is partially filled, providing

\footnotetext{
*Corresponding author: trudels@ucalgary.ca; OG current address: Quantum Nano-Scale Magnetics Laboratory, Advanced Science Institute, Riken, 2-1 Hirosawa, Wako 351-0198, Japan; JH current address: Department of Physics, VSB - Technical University of Ostrava, 17. listopadu 15, 70833 Ostrava-Poruba, Czech Republic
}

conducting electrons at the Fermi level $\varepsilon_{\mathrm{F}}$. The picture is quite different for the minority spin band (Fig. 1a), where $\varepsilon_{\mathrm{F}}$ is seen to lie within a band-gap, which is reminiscent of a semiconductor. At $\varepsilon_{\mathrm{F}}$, the spin polarization $P$, defined as

$$
P=\frac{N_{\text {maj }}-N_{\text {min }}}{N_{\text {maj }}+N_{\text {min }}}
$$

is $100 \%$, as the number of minority electrons $N_{\min }$ is strictly 0 . In other words, only electrons of one spin band participate in the electron transport properties of this material, or any half-metal. This property is highly desired for spin-dependent electron transport (spintronic) devices [13-15], and single-handedly accounts for the enthusiasm these materials have received in the past decade.

In addition to $\mathrm{NiMnSb}$ and $\mathrm{Co}_{2} \mathrm{FeSi}$, several other Heusler compounds have been predicted, from $a b$ initio calculations, to be half-metallic. Prominent are the $\mathrm{Co}_{2}$-based Heusler compounds, which gained a particular interest and became most widely studied in this field due to their high Curie temperatures, a requirement for integration into devices. However, despite the huge achievements that have been obtained in the last decade in magnetic tunnel junctions (MTJs) using $\mathrm{Co}_{2} M^{\prime} Z$ compounds as electrodes [15-22], an experimental proof of half-metallicity (i.e. robust $100 \%$ spin polarization at $\varepsilon_{\mathrm{F}}$ ) in these materials is still missing. While different mechanisms are thought to account for the suppression of the half-metallic state, this topic will not be covered in this Review. For an introduction to this topic, see for example the paper by Dowben and Skmoski [23]. Nonetheless, it is now clear that several $\mathrm{Co}_{2} M^{\prime} Z$ compounds do indeed support high spin polarizations higher than $85 \%$ [16, 21], much higher than the $3 d$ transition metals and their alloys, which typically have spin polarizations of $\sim 45 \%$ or lower $[24,25]$.

A parameter that will become key for future devices is the spin polarization at surfaces and interfaces, which is not guaranteed by bulk high spin polarization. At sur- 
(a) minority $\downarrow$

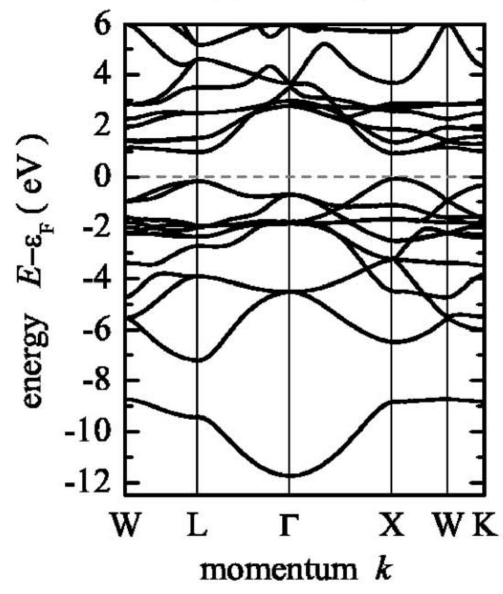

(b)

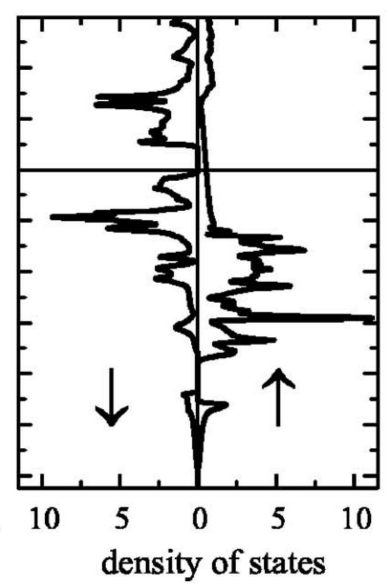

(c) majority $\uparrow$

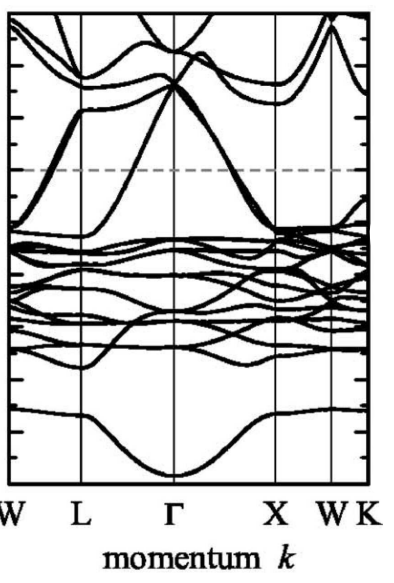

FIG. 1. Minority (a) and majority (c) band structure, and total density of states (b) of $\mathrm{Co}_{2} \mathrm{FeSi}$ calculated by $\mathrm{LDA}+U$. Reprinted figure with permission from Wurmehl, Fecher, Kandpal, Ksenofontov, Felser, Lin, and Morais, Phys. Rev. B, 72184434 (2005) [10]. Copyright (2005) by the American Physical Society. faces and interfaces the half-metallic property can be lost due to the introduction of surface and interface states at the Fermi level in the minority spin channel. For example, Galanakis has shown that the half-metallic character of $\mathrm{Co}_{2} \mathrm{MnSi}$ is lost at both Co- and MnSi-terminated surfaces [26]. Hashemifar and collaborators, however, have suggested that the half-metallicity of $\mathrm{Co}_{2} \mathrm{MnSi}$ can be preserved if the surface has the MnMn-termination [27], based on density functional calculations. Several other studies have looked into the spin polarization of the surfaces of $\mathrm{Co}_{2} \mathrm{MnSi}$ [26-33]. In addition to theoretical studies, reduction in surface magnetization has been observed, for example, by element-specific magnetic moment determination using x-ray magnetic circular dichroism $[34,35]$, suggesting a decrease in surface spin polarization.

The half-metallicity of the $\mathrm{Co}_{2} \mathrm{MnSi} / \mathrm{MgO}$ and $\mathrm{Co}_{2} \mathrm{MnSi} / \mathrm{GaAs}$ interfaces have also been theoretically studied [29-32]. The former is of particular importance for magnetic tunnel junctions, whereas the latter is considered for spin injection into semiconductors. Similar to the discussion of surfaces, interface states appear in the half-metallic gap because of the specific atomic environment of $\mathrm{Co}$ and $\mathrm{Mn}$ atoms in the topmost layer. These new states at the Fermi level can be filled by the electrons from the minority valence band through inelastic scattering processes (such as electron-phonon, electron-magnon and electron-electron scattering) thus making the spindown electrons contribute to the total conductivity of the interface [36]. Therefore, the polarization of the current passing through such an interface in a MTJ is reduced, and spin injection into a semiconductor becomes less efficient. Irrespective of the mechanism leading to the reduction of the spin polarization of surfaces and interfaces, this will become an increasingly important topic to address before achieving the full potential of half-metallic materials.

Apart from their spin polarization, other important magnetic properties that must be characterized and controlled are the magnetic anisotropy, magnetic exchange, and damping in cobalt-based full Heusler compounds. Magnetic anisotropies play a crucial role in determining the manner of magnetization reversal, and affect important properties such as the coercive field and remanence. The exchange interaction is closely related to the Curie temperature. Furthermore, processes involving magnons - such as electron-magnon interactions and the thermal excitation of magnons, pathways believed to strongly contribute to the decay of the spin polarization as a function of temperature - are intimately related to the energy of formation of magnons, accessed through the spin wave stiffness, another manifestation of the exchange interaction. Finally, dynamic processes cannot be discussed without introducing magnetic damping. Damping is also of paramount importance in spin-transfer-torque phenomena [37].

The main goal of this Topical Review is to provide an overview of the experimental studies concerned with the magnetic anisotropies, magnetic exchange, and damping properties of $\mathrm{Co}_{2}$-based Heusler compounds $\mathrm{Co}_{2} M^{\prime} Z$, or in other words, experimental manifestations of the spinspin and spin-orbit interactions. Given their technological impact, thin films will be emphasized. The structure of this Topical Review is as follows. After a brief introduction to the relevant magnetic and structural properties of $\mathrm{Co}_{2} M^{\prime} Z$ compounds (Section II), we will discuss the anisotropy and magnetization reversal (Section III), magnetic exchange (Section IV), and damping (Section V) in thin films of $\mathrm{CO}_{2} M^{\prime} Z$ compounds.

\section{BASIC STRUCTURAL AND MAGNETIC PROPERTIES OF $\mathrm{CO}_{2}$-BASED HEUSLER COMPOUNDS}

We begin our overview by rapidly introducing the salient structural and magnetic properties of Heusler compounds. For more details, the reader is directed to the textbook by Kübler [38], or the reviews by Galanakis $[39,40]$, which deal with half-metallicity, Slater-Pauling 


\begin{tabular}{|c|c|c|c|c|}
\hline Compound & $\begin{array}{c}N_{\mathrm{V}} \\
\left(\mathrm{e}^{-} / \text {f.u. }\right)\end{array}$ & Structure & $\begin{array}{c}a_{\exp }{ }^{\star} \\
(\AA)\end{array}$ & Ref. \\
\hline $\mathrm{Co}_{2} \mathrm{Cr}_{0.6} \mathrm{Fe}_{0.4} \mathrm{Al}$ & 27.8 & B2 & 5.737 & {$[44]$} \\
\hline $\mathrm{Co}_{2} \mathrm{MnAl}$ & 28 & B2 & 5.756 & [45] \\
\hline $\mathrm{Co}_{2} \mathrm{MnSi}$ & 29 & $\mathrm{~L} 2_{1}$ & 5.654 & [45] \\
\hline $\mathrm{Co}_{2} \mathrm{MnGe}$ & 29 & $\mathrm{~L} 2_{1}$ & 5.743 & [45] \\
\hline $\mathrm{Co}_{2} \mathrm{FeAl}$ & 29 & B2 & 5.730 & [44] \\
\hline $\mathrm{Co}_{2} \mathrm{FeGa}$ & 29 & $\mathrm{~L} 2_{1}$ & 5.741 & {$[46]$} \\
\hline $\mathrm{Co}_{2} \mathrm{FeSi}$ & 30 & $\mathrm{~L} 2_{1}$ & 5.640 & [10] \\
\hline
\end{tabular}

* See [47] and references therein for a more

extensive compilation.

TABLE I. Experimental bulk lattice parameters of selected $\mathrm{Co}_{2} M^{\prime} Z$ Heusler compounds

behaviour, and the electronic structure of Heusler compounds.

\section{A. Structure and disorder}

Heusler compounds with the general formula $\mathrm{Co}_{2} M^{\prime} Z$ crystallize in the $\mathrm{L} 2{ }_{1}$ structure $(F m \overline{3} m$ space group) which is shown in Fig. 2a. The cubic unit cell consists of four interpenetrating $f c c$ sublattices, two of which are occupied by Co atoms, and the other two by the $M^{\prime}$ and $Z$ atoms, respectively. The two Co sublattices are positioned at the $8 c$ Wyckoff positions $\left(\frac{1}{4}, \frac{1}{4}, \frac{1}{4}\right)$ of the cubic unit cell, while the $M^{\prime}$ and $Z$ atoms occupy the $4 a(0,0,0)$ and $4 b\left(\frac{1}{2}, \frac{1}{2}, \frac{1}{2}\right)$ positions, respectively (note that one often finds in the literature equivalent images where the structures has been shifted by $\left(\frac{1}{4}, \frac{1}{4}, \frac{1}{4}\right)$ so that the cobalt occupies the corners of the unit cell). The L2 1 structure represents the most ordered phase of the $\mathrm{Co}_{2}$-based Heusler compounds. However, various degrees of disorder can exist for a given chemical composition, in the form of interchange of atoms between different sublattices. If the combined $(0,0,0)$ and $\left(\frac{1}{2}, \frac{1}{2}, \frac{1}{2}\right)$ sites are randomly occupied by $M^{\prime}$ and $Z$ with an equal probability, the structure is referred to as the B2 structure (Fig. 2b). The interchange of atoms in Co and $M^{\prime}$ sublattices results in the $\mathrm{DO}_{3}$ structure (Fig. 2c). A random occupation of all four sublattices leads to a structure which is called the A2 structure, and shown in Fig. 2d [41-43]. See Bacon and Plant [41] for a further description of ordering in Heusler compounds.

Experimentally, information about the structural properties, such as lattice parameters and the type of disorder present in the samples, is obtained using a variety of methods, such as x-ray [48-50] and neutron [51] diffraction, anomalous x-ray diffraction [52-54], extended x-ray absorption fine structure (EXAFS) [49, 51], and nuclear magnetic resonance (NMR) [55]. Structural information concerning atomic disorder utilizing both x-ray and neutron scattering is provided by the analysis of relative in-

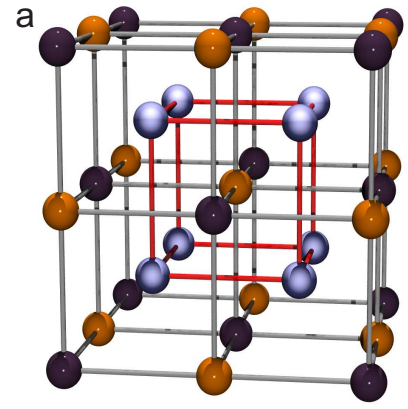

$\mathrm{L} 2$ : $\mathrm{CO}_{2} \mathrm{M} \mathrm{Z} Z$

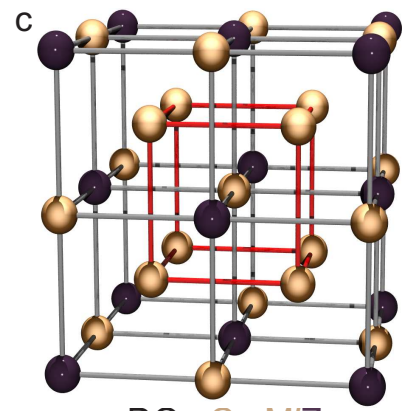

$\mathrm{DO}_{3}: \mathrm{Co}_{2} \mathrm{M} \mathrm{M}^{\prime} \mathrm{Z}$

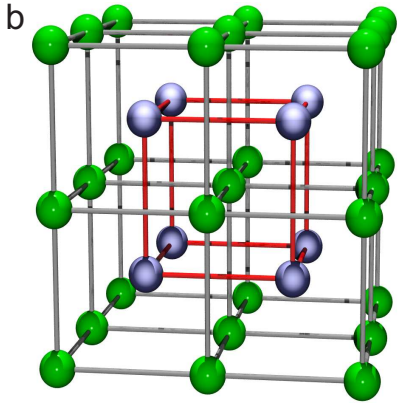

B2: $\mathrm{CO}_{2} \mathrm{M}^{\prime} \mathrm{Z}$

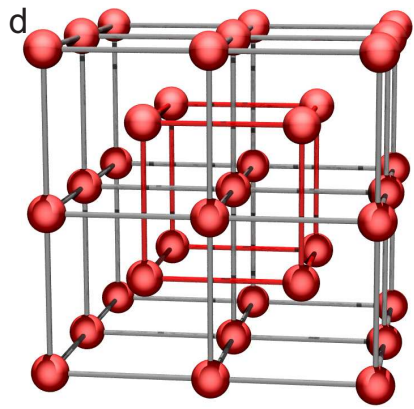

A2: $\mathrm{Co}_{2} \mathrm{M}^{\prime} \mathrm{Z}$
FIG. 2. $\mathrm{L}_{1}$ (a), B2 (b), $\mathrm{DO}_{3}$ (c), and A2 (d) ordered structures for $\mathrm{Co}_{2} M^{\prime} Z$ Heusler compounds. The lattice consists of four interpenetrating $f c c$ sublattices, two of which are occupied by Co, the other two by $M^{\prime}$ and $Z$ atoms, respectively. (Colour online)

tensities of superstructure reflections. While less widely available than x-ray diffraction, NMR was demonstrated to a be a very powerful method for the characterization of atomic disorder [56-58]. The bulk lattice parameters of some of the more commonly encountered $\mathrm{Co}_{2}$-based Heusler compounds are listed in Table I.

In order to obtain Heusler films with the well-ordered $\mathrm{L} 2{ }_{1}$ structure, high temperature annealing is usually required since the $\mathrm{B} 2 / \mathrm{L} 21$ phase transition in the bulk occurs at about $1000 \mathrm{~K}$ in most of the $\mathrm{Co}_{2}$-based Heusler compounds [59-61], while this transition occurs around $800 \mathrm{~K}$ in thin films [62, 63]. It is interesting to note, however, that the L2 1 phase is not always the most stable one from the thermodynamic point of view. For example, $\mathrm{Co}_{2} \mathrm{Cr}_{0.6} \mathrm{Fe}_{0.4} \mathrm{Al}$ preferably crystallizes in the $\mathrm{B} 2$ structure [64] while the $\mathrm{L} 22_{1}$ structure is more stable for $\mathrm{Co}_{2} \mathrm{MnSi}$ and $\mathrm{Co}_{2} \mathrm{FeSi}$. Referring to Table I, it can generally be observed that Si-bearing compounds tend to crystallize in the $\mathrm{L} 2{ }_{1}$ structure, while $\mathrm{Al}$ counterparts adopt the B2 structure. The origin of this most likely stems from the difference in bonding between $\mathrm{Si}, \mathrm{Al}$, and the transition metals [47].

As will be discussed below, important magnetic properties, such as the saturation magnetization, spin wave stiffness, magnetocrystalline anisotropy, and Gilbert damping constant are affected by the level of ordering. Unfortunately, a rigorous determination of the order 


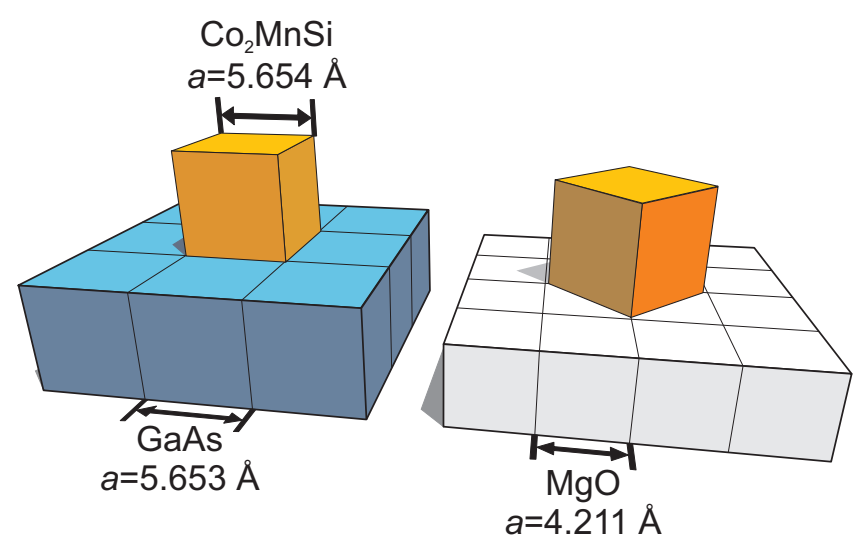

FIG. 3. Epitaxial relationships between $\mathrm{Co}_{2} \mathrm{MnSi}$ and $\operatorname{GaAs}(001)$ (left) and $\mathrm{MgO}(001)$ (right). (Colour online)

does not always accompany the characterization of these quantities, and so the establishment of reliable structureproperty relationships is still challenging.

For high-quality spintronic devices and significant structure-relationships to be established, well-defined samples are required. As such, most studies highlighted in this Topical Review deal with high-quality thin films epitaxially grown using high-vacuum deposition methods. By far, the two most widely used substrates are $\mathrm{GaAs}(001)$ and $\mathrm{MgO}(001)$. In general the III-V semiconductors are substrates amenable to the growth of Heusler compounds. If one takes the $\mathrm{Co}_{2} M^{\prime} Z$ structure and leaves the $M^{\prime}$ and one of the Co sublattices empty, one has formed the zincblende structure adopted by III-V semiconductors. While for $\mathrm{Co}_{2}$-based Heuslers GaAs is better lattice-matched, InP is, for example, well latticematched to compounds such as $\mathrm{Ni}_{2} \mathrm{MnGa}$, while InAs matches to $\mathrm{Rh}_{2} \mathrm{MnAl}$ [65]. When using $\operatorname{GaAs}(001)$ for the growth of $\mathrm{Co}_{2} M^{\prime} Z$, a cube-on-cube epitaxial relationship is present, as shown on the left side of Fig. 3 where a unit cell of $\mathrm{Co}_{2} \mathrm{MnSi}$ is shown on $\mathrm{GaAs}(001)$. In the case of $\mathrm{MgO}(001)$, the lattice cell parameter $a$ is too small to accommodate a $\mathrm{Co}_{2} M^{\prime} Z$ compound in the same fashion as GaAs. However, if the $\mathrm{Co}_{2} M^{\prime} Z$ cube edge grows at an angle of $45^{\circ}$ with respect to an in-plane $\mathrm{MgO}\langle 100\rangle$ direction, a good lattice match is obtained (see Fig. 3, right hand side), enabling epitaxial growth. This is particularly important towards the implementation of magnetic tunnelling junctions (MTJs) taking advantage of coherent tunnelling through a single-crystal $\mathrm{MgO}$ barrier $[66,67]$.

\section{B. Half-metallicity and origin of the minority spin gap}

The expression "half-metal" was coined by de Groot et al., based on band structure calculations for the NiMnSb half-Heusler compound [11]. This term is employed to describe material systems with an asymmetry
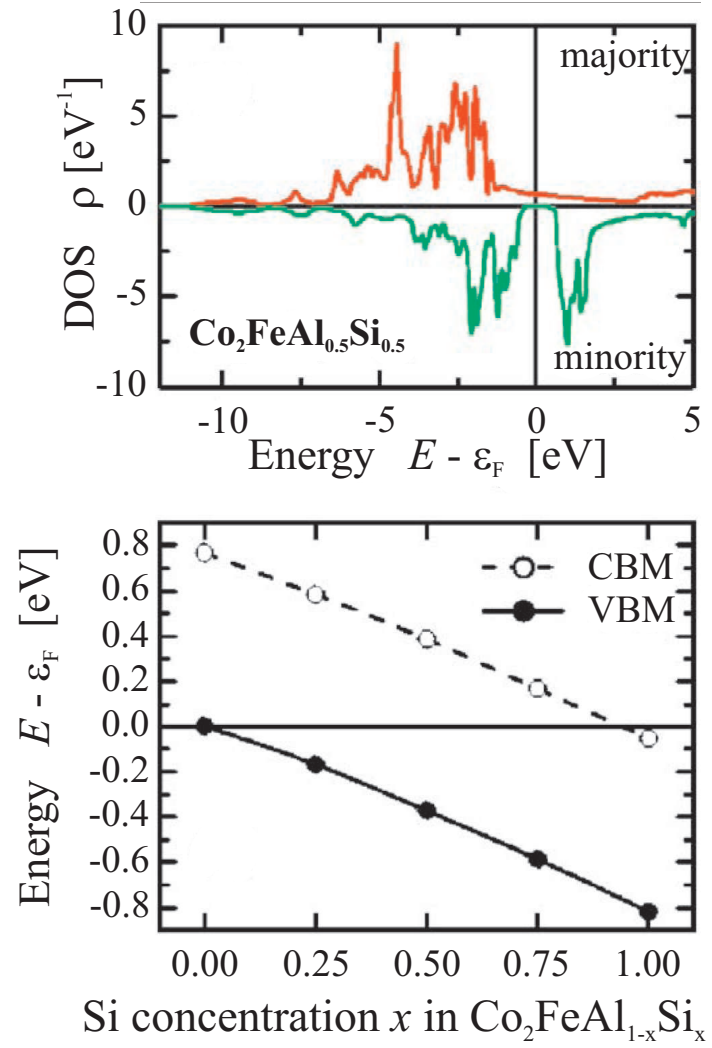

FIG. 4. Top shows the density of states of $\mathrm{Co}_{2} \mathrm{Fe}_{0.5} \mathrm{Al}_{0.5} \mathrm{Si}$ as calculated by means of $\mathrm{LDA}+U$. Bottom shows the gap in the minority states with respect to the Fermi energy $\varepsilon_{\mathrm{F}}$. Compared are the positions of the valence band maximum (VBM) and the conduction band minimum (CBM). Reprinted with permission from [76]. Copyright 2007, American Institute of Physics. (Colour online)

of the spin-split band structure: while the majority spin band has a metallic behaviour (i.e. a non-vanishing density of states (DOS) at the Fermi level), the minority electrons exhibit a semiconducting character (i.e. a band gap around the Fermi level) (Fig. 1).

As mentioned above, several $\mathrm{Co}_{2}$-based Heusler compounds were predicted to be half-metals. First theoretical evidence of the half-metallicity in $\mathrm{Co}_{2} \mathrm{MnSi}$ was given by Ishida et al. [28], and was later confirmed by various authors [47, 68-70]. Additionally, half-metallic behaviour has been predicted for $\mathrm{Co}_{2} \mathrm{FeSi}[10,71], \mathrm{Co}_{2} \mathrm{FeAl}[47,71]$, and $\mathrm{Co}_{2} \mathrm{MnGe}[39,68,72,73]$. Kübler et al. reported for the first time the existence of a band gap for the minority electrons in the related $\mathrm{Co}_{2} \mathrm{MnAl}$ Heusler compound [74]. In this compound, however, the Fermi energy falls into the upper tail of the valence band and a nonvanishing spin-down DOS is found at the Fermi level. More recent calculations showed similar results for the electronic structure of this material $[47,69,75]$. Nevertheless, $\mathrm{Co}_{2} \mathrm{MnAl}$ remains an interesting candidate for applications because its expected spin polarization is still comparatively high.

The electronic structure of $\mathrm{Co}_{2} M^{\prime} Z$ Heusler com- 
pounds and their physical properties can be tuned by alloying with a fourth element, which provides a new way of designing materials with tailored characteristics $[60,71,77-79]$. In the quaternary compounds, the atoms in one of the sublattices are partially substituted by atoms of another element. $\mathrm{Co}_{2} \mathrm{Cr}_{0.6} \mathrm{Fe}_{0.4} \mathrm{Al}$, obtained from $\mathrm{CO}_{2} \mathrm{CrAl}$ by doping with $\mathrm{Fe}$ atoms, represents one of the most prominent examples in this class of Heusler compounds. In this case, a partial substitution of $\mathrm{Cr}$ for Fe resulted in a large magnetoresistance effect of about $30 \%$ in polycrystalline powder compacts, which was not observed for the initial ternary compound $\mathrm{Co}_{2} \mathrm{CrAl}$ [80]. Miura et al. investigated in detail the complete substitutional series $\mathrm{Co}_{2} \mathrm{Cr}_{1-x} \mathrm{Fe}_{x} \mathrm{Al}$ from the theoretical point of view [64]. Similar to the case $\mathrm{Co}_{2} \mathrm{MnAl}$, a non-vanishing DOS is expected in the minority band of $\mathrm{Co}_{2} \mathrm{Cr}_{0.6} \mathrm{Fe}_{0.4} \mathrm{Al}$ according to these calculations, for both the B2 and $\mathrm{L} 2{ }_{1}$ ordered phases. More recently, the $\mathrm{Co}_{2} \mathrm{FeAl}_{1-x} \mathrm{Si}_{x}$ series has attracted much attention, as band structure calculations $(\mathrm{LDA}+U)$ have shown that the position of the Fermi level $\varepsilon_{\mathrm{F}}$ in the band gap is tuned by composition $[76,79]$. For $\mathrm{Co}_{2} \mathrm{FeAl}(x=0), \varepsilon_{\mathrm{F}}$ lies just above the valence band. Introducing valence electrons by replacing $\mathrm{Al}$ by $\mathrm{Si}$ raises the Fermi level. For $\mathrm{Co}_{2} \mathrm{FeSi}(x=1) \varepsilon_{\mathrm{F}}$ lies just below the conduction band. For $x=0.5, \varepsilon_{\mathrm{F}}$ lies in the middle of the minority spin band gap (see Fig. 4). As such, effects which lead to the reduction of the spin polarization due to smearing of the band edges should be greatly minimized. A recent report from Shan and collaborators [16] on the tunnelling magnetoresistance of $\mathrm{Co}_{2} \mathrm{Fe}_{0.5} \mathrm{Al}_{0.5} \mathrm{Si} / \mathrm{MgAl}_{2} \mathrm{O}_{x} / \mathrm{CoFe}$ junctions seems to support this contention.

In 2002 Galanakis and collaborators proposed a model describing the origin of the minority band gap [69]. For example, the minority spin band structure of $\mathrm{Co}_{2} \mathrm{MnGe}$ is shown in Fig. 5a [69]. The observed symmetry and energy ordering of the bands at the $\Gamma$-point can be understood using a group-theoretical analysis. First, one considers hybridization of the Co $3 d$ states $\left(t_{2 \mathrm{~g}}\right.$ and $\left.e_{\mathrm{g}}\right)$ between Co on distinct fcc lattices (Fig. 5b)(states with an $e$ representation are doubly degenerate [the $d_{x^{2}-y^{2}}$ and $d_{z^{2}}$ states for $e_{g}$ ], while states transforming with the $t$ representation are triply degenerate [the $d_{x y}, d_{x z}$, and $d_{y z}$ states for $\left.\left.t_{2 g}\right]\right)$. The symmetry-adapted states of each Co mix, yielding (in order of increasing energy) the $e_{\mathrm{g}}$, $t_{2 \mathrm{~g}}, t_{1 \mathrm{u}}$, and $e_{\mathrm{u}}$ states. These hybridized states are then mixed with the $M^{\prime} 3 d$ states Fig. 5(c). The symmetrymatched $\operatorname{Co}\left(e_{\mathrm{g}}\right)$ and $M^{\prime}\left(e_{\mathrm{g}}\right)$ states mix, and the same occurs for $\mathrm{Co}\left(t_{2 \mathrm{~g}}\right)-M^{\prime}\left(t_{2 \mathrm{~g}}\right)$ states. However, on the $M^{\prime}$ there are no states with symmetry corresponding to the Co $t_{1 \mathrm{u}}$ and $e_{\mathrm{u}}$ states. As such, these states remain nonbonding, and will not hybridize with $d$ states of $M^{\prime}$. The Fermi level lies in the energy gap between these Co $t_{1 \mathrm{u}}$ and $e_{\mathrm{u}}$ states.

In the above discussion the main group $Z$ atom has been completely disregarded. This is because its lowlying $s$ and $p$ states do not directly contribute to the formation of the minority band gap. However, as these

\begin{tabular}{lccc}
\hline \hline Compound & $\begin{array}{c}m_{\mathrm{SP}} \\
\left(\mu_{\mathrm{B}} / \text { f.u. }\right)\end{array}$ & $\begin{array}{c}m_{\text {exp }}{ }^{\star} \\
\left(\mu_{\mathrm{B}} / \text { f.u. }\right)\end{array}$ & $\begin{array}{c}T_{\mathrm{C}} \\
(\mathrm{K})\end{array}$ \\
\hline $\mathrm{Co}_{2} \mathrm{Cr}_{0.6} \mathrm{Fe}_{0.4} \mathrm{Al}$ & 3.48 & $3.30[80]$ & $750[80]$ \\
$\mathrm{Co}_{2} \mathrm{MnAl}$ & 4.00 & $4.04[81]$ & $693[45,81]$ \\
$\mathrm{Co}_{2} \mathrm{MnSi}$ & 5.00 & $4.96[82]$ & $985[45,82]$ \\
$\mathrm{Co}_{2} \mathrm{MnGe}$ & 5.00 & $4.84[82]$ & $905[45,82]$ \\
$\mathrm{Co}_{2} \mathrm{FeAl}$ & 5.00 & $4.96[81]$ & $\approx 1000$ \\
$\mathrm{Co}_{2} \mathrm{FeGa}$ & 5.00 & $5.15[82]$ & $>1100[82]$ \\
$\mathrm{Co}_{2} \mathrm{FeSi}$ & 6.00 & $6.00[10]$ & $1100[10]$ \\
\hline \hline
\end{tabular}

* See [47] and references therein for a more

extensive compilation.

TABLE II. Slater-Pauling and experimental and saturation magnetization and experimental Curie temperatures

states contribute to the total number of occupied and empty states, the $Z$ atom is important for the position of the Fermi level within the minority band gap as explained above for $\mathrm{Co}_{2} \mathrm{FeAl}_{1-x} \mathrm{Si}_{x}$. Moreover, the $s$ and $p$ states have been shown to play an important role for the distribution of electrons in the various symmetry distinguished states $\left(t_{2 g}\right.$ and $\left.e_{g}\right)$ at Co and $M^{\prime}$ sites [47].

\section{Slater-Pauling behaviour and magnetic moments}

The origin of the ferromagnetic behaviour in $\mathrm{Co}_{2} M^{\prime} Z$ is rather complicated, and is still one of the most interesting problems in modern magnetism.

One of the most tell-tale characteristics of the halfmetallic Heusler compounds is their integer magnetic moments. To understand this behaviour, we again refer to the origin of the minority spin gap and Fig. 5. As was discussed above, $\mathrm{Co}_{2} M^{\prime} Z$ Heusler compounds have eight occupied minority $d$ states per unit cell, namely (in order of increasing energy) the $e_{g}, t_{2 g}$, and $t_{1 u}$ bands, the latter lying just below the Fermi level (see Fig. 5b) [39, 69]. Apart from these eight $d$ bands, there are one $s$ and three $p$ bands, which are much lower in energy $(\sim 5-6 \mathrm{eV}$ below $\left.\varepsilon_{\mathrm{F}}\right)$ and do not contribute to the formation of the band gap. Thus, in total the $\mathrm{Co}_{2}$-based Heusler compounds possess 12 occupied minority states $N_{\text {min }}$ per unit cell. The total magnetic moment $m$ (in units of Bohr magnetons $\mu_{\mathrm{B}}$ per formula unit f.u.) is given by the number of excess majority electrons with respect to minority electrons, i.e.

$$
m=N_{\text {maj }}-N_{\text {min }} .
$$

Introducing

$$
N_{\mathrm{V}}=N_{\text {maj }}+N_{\text {min }}
$$

as the total number of valence electrons in the unit cell, and using $N_{\min }=12$ as discussed above, the magnetic 

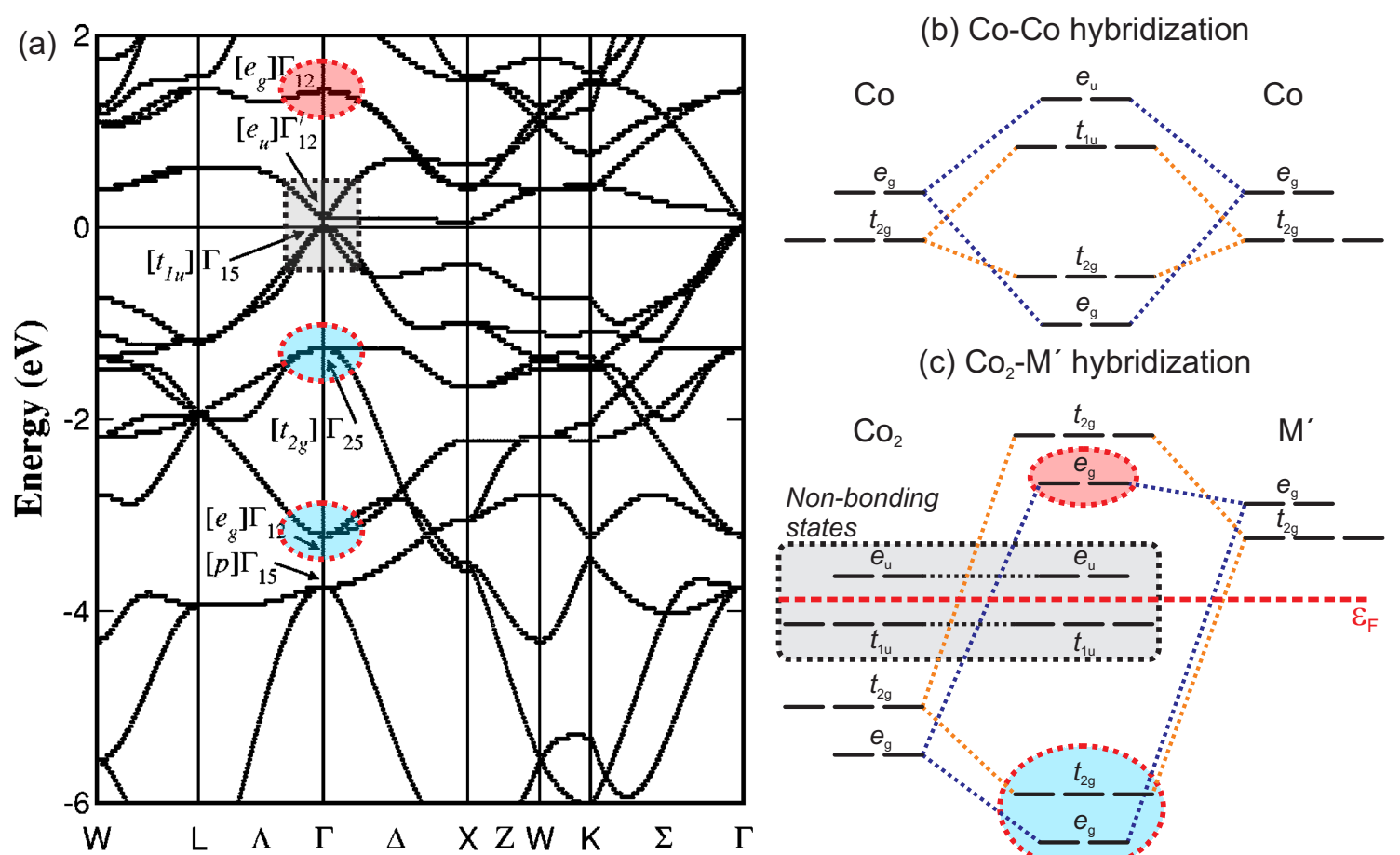

(c) $\mathrm{Co}_{2}-\mathrm{M}^{\prime}$ hybridization

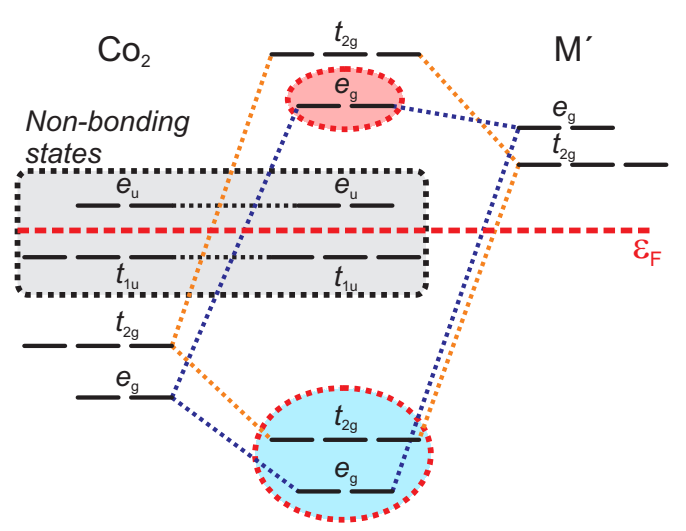

FIG. 5. (a) Spin-down band structure of the $\mathrm{Co}_{2} \mathrm{MnGe}$ compound. Adapted figure with permission from Galanakis, Dederichs, Papanikolaou, Phys. Rev. B 66174429 (2002) [69]. Copyright (2002) by the American Physical Society. (b) Proposed Co-Co hybridization (from distinct $f c c$ sublattices). (c) Proposed $\mathrm{Co}_{2}-M^{\prime}$ hybridization. The highlighted states are also highlighted in (a). Three $p$ and one $s$ states are missing (lower in $\mathcal{E}$ ), to provide the 12 minority states. (Colour online)

moment $m$ of the $\mathrm{Co}_{2} M^{\prime} Z$ Heusler compounds should obey the rule

$$
m=N_{\mathrm{V}}-24 \text {. }
$$

This is known as the generalized Slater-Pauling rule [83], which is an analogue to the Slater-Pauling behaviour of the binary transition metal alloys $[38,84-86]$. This equation places the Heusler compounds into the region of systems with localized magnetic moments [38, 83, 87]. Since in the $\mathrm{Co}_{2} M^{\prime} Z$ Heusler compounds $N_{\mathrm{V}}$ is an integer number, an integer magnetic moment follows from this rule. Note that while Eq. 4 is still valid for quaternary compounds, an integer magnetic moment is no longer expected due to the non-integer site occupancies.

\section{Formation of local magnetic moments}

A microscopic mechanism resulting in the formation of localized magnetic moments in the $\mathrm{Co}_{2}$-based Heusler compounds was first given by Kübler and collaborators [74].

It was later recognized that the magnetic moment mainly resides on $\mathrm{Co}$ and $M^{\prime}$, while the moment on $Z$ atoms is negligible. Nevertheless, the $Z$ atom plays an important role for the formation of the individual magnetic moments at Co and $M^{\prime}$ sites [47]. For example, in $\mathrm{Co}_{2} M^{\prime} Z\left(M^{\prime}=\mathrm{Mn}\right.$ or $\mathrm{Fe} ; Z=\mathrm{Al}$ or $\left.\mathrm{Si}\right)$ replacement of
Al by Si results in an increase of localized magnetic moments on both Co and $\mathrm{Mn}$ (or Fe) sites, which is due to the filling of the majority bands with an additional electron. The small moments found at $s p$ sites are mainly due to a polarization of these atoms by the surrounding, magnetically active Co and $M^{\prime}$ atoms. Co atoms bear a larger magnetic moment in the Si compounds with respect to the $\mathrm{Al}$ compounds. The reason for this behaviour is a stronger bonding interaction between $\mathrm{Co}$ and $\mathrm{Si}$ as compared to the Co-Al case due to the higher electronegativity of $\mathrm{Si}[47]$.

\section{E. Exchange interaction between magnetic moments}

Recently, extensive theoretical studies have been reported by Kurtulus et al. and Şaşioğlu et al. elucidating the coupling mechanism of the localized magnetic moments in $\mathrm{Co}_{2}$-based Heusler compounds $[70,88]$. In the case of $\mathrm{Co}_{2} \mathrm{MnSi}$, both groups found that the interaction between the nearest $\mathrm{Mn}$ and Co atoms provides the leading interaction for the stabilization of the ferromagnetism in this system. The magnetic moments localized at Mn and Co sites are coupled ferromagnetically to each other via the direct exchange coupling mechanism. This is in contrast to the mechanism proposed by Kübler in his early theoretical investigations where the Mn-Mn inter- 
action was proposed to be responsible for the appearance of ferromagnetism in $\mathrm{Co}_{2} \mathrm{MnSi}$ [74]. In addition to the leading $\mathrm{Co}-\mathrm{Mn}$ interaction, the $\mathrm{Mn}-\mathrm{Mn}$ interaction and the coupling of the nearest Co magnetic moments located on different Co sublattices were found to be important for the stabilization of ferromagnetism in $\mathrm{Co}_{2} \mathrm{MnSi}$. These contributions, however, are about one order in magnitude smaller than the leading Co-Mn interaction. Although the $\mathrm{Mn}$ atoms have a larger magnetic moment, the ferromagnetic interaction between Co atoms is stronger, which is rationalized by a smaller distance between Co atoms, as compared to that between the Mn atoms. Due to a large Mn-Mn separation ( $>4 \AA$ ) there is no significant direct exchange interaction. The coupling of $\mathrm{Mn}$ moments is believed to be mediated by a RKKY interaction, since the characteristic oscillations of the exchange parameter are found in dependence of the interatomic distance. Co atoms positioned in the same sublattice exhibit a similar behaviour and contribute as well to the stabilization of ferromagnetism in the $\mathrm{Co}_{2} \mathrm{MnSi}$ Heusler compound. Results recently published by Thoene et al. are in agreement with the above described coupling mechanism in $\mathrm{Co}_{2} \mathrm{MnSi}$ [89].

Not all $\mathrm{Co}_{2} M^{\prime} Z$ compounds were thoroughly investigated. However, given their similar structural and electronic properties, the exchange mechanisms in these materials can reasonably be expected to be similar to those described for the $\mathrm{Co}_{2} \mathrm{MnSi}$ Heusler compound. This assumption is supported by the results of investigations carried out for $\mathrm{Co}_{2} \mathrm{CrAl}$ [70], which showed the same coupling mechanisms are responsible for the stabilization of the ferromagnetic state in this compound.

The strength of the exchange interaction is found to be dependent on the lattice constant. In particular, the lattice contraction leads to an enhancement of intra- and intersublattice exchange constants [70, 88]. Therefore, an increase of the exchange constants is expected in the investigated series of $\mathrm{Co}_{2}$-based Heusler compounds for those with a smaller $a$ value.

\section{F. Curie temperature}

Due to the robustness of the ferromagnetic order, which arises from the strong inter- and intrasublattice exchange interactions, the $\mathrm{Co}_{2}$-based Heusler compounds exhibit remarkably high Curie temperatures $T_{\mathrm{C}}$ (Table II). Their $T_{\mathrm{C}}$ values are comparable to those of pure $3 d$ metals. When considered as a function of the number of valence electrons, the $T_{\mathrm{C}}$ of $\mathrm{Co}_{2}$-based Heusler compounds show a linear dependence similar to the SlaterPauling behaviour discussed above [90]. The same trend was reported for $T_{\mathrm{C}}$ values as a function of the magnetic moment [87].

The origin of the linear dependence between $T_{\mathrm{C}}$ and $N_{\mathrm{V}}$ was carefully investigated by Kübler et al. by means of $a b$ initio calculations [90] using the frozen-magnon approach [91]. The authors estimate the Curie temperature using the expression

$$
k_{\mathrm{B}} T_{\mathrm{C}}=\frac{2}{3} \sum_{\tau} \mathcal{L}_{\tau}^{2}\left[\frac{1}{N} \sum_{\mathbf{q} n} \frac{1}{j_{n}(\mathbf{q})}\right]^{-1}
$$

where $\mathcal{L}_{\tau}$ is the local magnetic moment at site $\tau$ and $j_{n}(\mathbf{q})$ are three exchange functions, which describe the inter- and intrasublattice exchange interactions. The expression in the square brackets in Eq. (5) represents an exchange average. It was found that $T_{\mathrm{C}}$ is determined by both the magnetic moments and the average exchange value. The proportional relation between $T_{\mathrm{C}}$ and the number of valence electrons is the result of these combined factors. The same interplay is responsible for the linear trend found for $T_{\mathrm{C}}$ and the magnetic moment [87].

\section{MAGNETIC ANISOTROPY}

The study of the magnetization reversal or the excitation of spin waves, and their dependence on the relative orientation between magnetization and crystallographic orientations, allow one to study the anisotropy of the magnetic properties of thin films. This anisotropy can stem from several effects, such as shape anisotropy due to geometry-dependent demagnetization factors in structured elements (which in thin films coerces the magnetization to lie within the plane of the thin film), stress (external or due to strain from epitaxial mismatch), or the magneto-crystalline anisotropy due to spin-orbit coupling [92].

The spin-orbit interaction, coupling spin and orbital angular momenta $\mathbf{S}$ and $\mathbf{L}$, can be expressed for localized moments in a general form as

$$
\mathcal{H}_{\mathrm{SO}}=\xi(r) \mathbf{L} \cdot \mathbf{S}
$$

and the magneto-crystalline anisotropy is given by the difference in energy between the hard and easy directions of magnetization (i.e. the anisotropy of the spin-orbit energy),

$$
\begin{aligned}
\Delta \varepsilon_{\mathrm{SO}} & =\left\langle\mathcal{H}_{\mathrm{SO}}\right\rangle_{\text {hard }}-\left\langle\mathcal{H}_{\mathrm{SO}}\right\rangle_{\text {easy }} \\
& =\langle\xi(r)\rangle\left[\langle\mathbf{L} \cdot \mathbf{S}\rangle_{\text {hard }}-\langle\mathbf{L} \cdot \mathbf{S}\rangle_{\text {easy }}\right]
\end{aligned}
$$

where $\langle\xi(r)\rangle$ is the spin-orbit coupling constant [93]. As can be seen in Eq. (7), the orientational dependence stems from the directional character of orbital moments L through anisotropic bonding within the crystal lattice.

Because of their cubic structure, Heusler compounds have long been considered as materials with completely quenched orbital moments. Recently, however, noticeable orbital moments were found in x-ray magnetic circular dichroism (XMCD) studies for $\mathrm{Co}_{2} \mathrm{FeAl}$ and $\mathrm{Co}_{2} \mathrm{Cr}_{0.6} \mathrm{Fe}_{0.4} \mathrm{Al}[94,95], \mathrm{Co}_{2} \mathrm{MnGe}$ [96], $\mathrm{Co}_{2} \mathrm{MnAl}$ and $\mathrm{Co}_{2} \mathrm{MnSi}$ [97], and $\mathrm{Co}_{2} \mathrm{FeSi}$ [10], leading to an increased interest in the orbital magnetism of $\mathrm{Co}_{2}$-based Heusler compounds from the theoretical point of view. Several 
computational studies were dedicated to the determination of element-specific magnetic moments in $\mathrm{Co}_{2} M^{\prime} Z$ compounds $[98,99]$. However, none of them could give a satisfactory agreement with the experimental results: the calculated orbital moments are usually 2-4 times smaller compared to the experimental values. The discrepancy is thought to arise from difficulties in a proper treatment of the orbital magnetism in ab initio calculations on the one hand, and systematic artifacts in the analysis of XMCD data on the other. Since the magnetocrystalline anisotropy is directly related to the orbital magnetic moments, a larger anisotropy might therefore be expected in $\mathrm{Co}_{2} M^{\prime} Z$ compounds.

In practice, the anisotropy is phenomenologically described in real systems by the anisotropy constant $K$. Experimentally, its value can be accessed through Brillouin light scattering (BLS) spectroscopy, ferromagnetic resonance (FMR), magneto-optical Kerr effect (MOKE) magnetometry, or torque magnetometry. To the best of our knowledge, the latter method has not been applied to the study of thin films of Heusler compounds. Examples of the other three methods will be provided below. Procedures on how BLS spectroscopy and FMR are used to determine anisotropies are offered, for example, by Hillebrands (BLS) [100] and Yilgin and Aktas (FMR) [101]. Finally, the study of anisotropy, i.e. the relationship between the magnetization and well-defined directions in the sample, requires high-quality single-crystal films grown in an epitaxial fashion. All the examples below treat such epitaxially grown Heusler compounds on GaAs or $\mathrm{MgO}$ (c.f. Fig. 3 above for the epitaxial relations in these two systems).

In this section, a few case studies will be presented. For those particularly interested in quantitative studies, a compilation of anisotropy constants for various compounds is presented in Table III, along with the relevant references to the primary literature. The reader should refer to the cited literature for complete descriptions of the samples and models used.

Due to limited space, it is impractical to review all studies relating to the anisotropy of $\mathrm{Co}_{2} M^{\prime} Z$ compounds. Instead, a few selected case studies are highlighted below.

Case study 1: Cubic anisotropy and magnetization reversal in $\mathrm{Co}_{2} \mathrm{Cr}_{0.6} \mathrm{Fe}_{0.4} \mathrm{Al}$

Given the cubic symmetry of Heusler compounds, a fourfold symmetry is expected when studying the orientation dependence of the magnetic properties of [100]oriented films. Such is the behaviour that was observed for an $80 \mathrm{~nm}$ thick $\mathrm{Co}_{2} \mathrm{Cr}_{0.6} \mathrm{Fe}_{0.4} \mathrm{Al}(100)$ film grown on Cr-buffered $\mathrm{MgO}(100)$, as is shown in Fig. 6a [115]. Here, a polar plot of the coercive field $H_{\mathrm{c}}$ determined by MOKE magnetometry as a function of in-plane angle is shown. The first salient feature is the clear fourfold symmetry of the result. The $\langle 110\rangle$ directions are identified as the easy axes of direction from their higher coercive field, while the $\langle 100\rangle$ directions are apparently the hard axes. Which brings up the second salient feature of Fig. 6a, the sharp peaks in $H_{\mathrm{c}}$ for the $\langle 100\rangle$ directions. To understand the
$\mathrm{MgO}(001) / \mathrm{Cr}(8 \mathrm{~nm}) / \mathrm{CCFA}(80 \mathrm{~nm})$

a
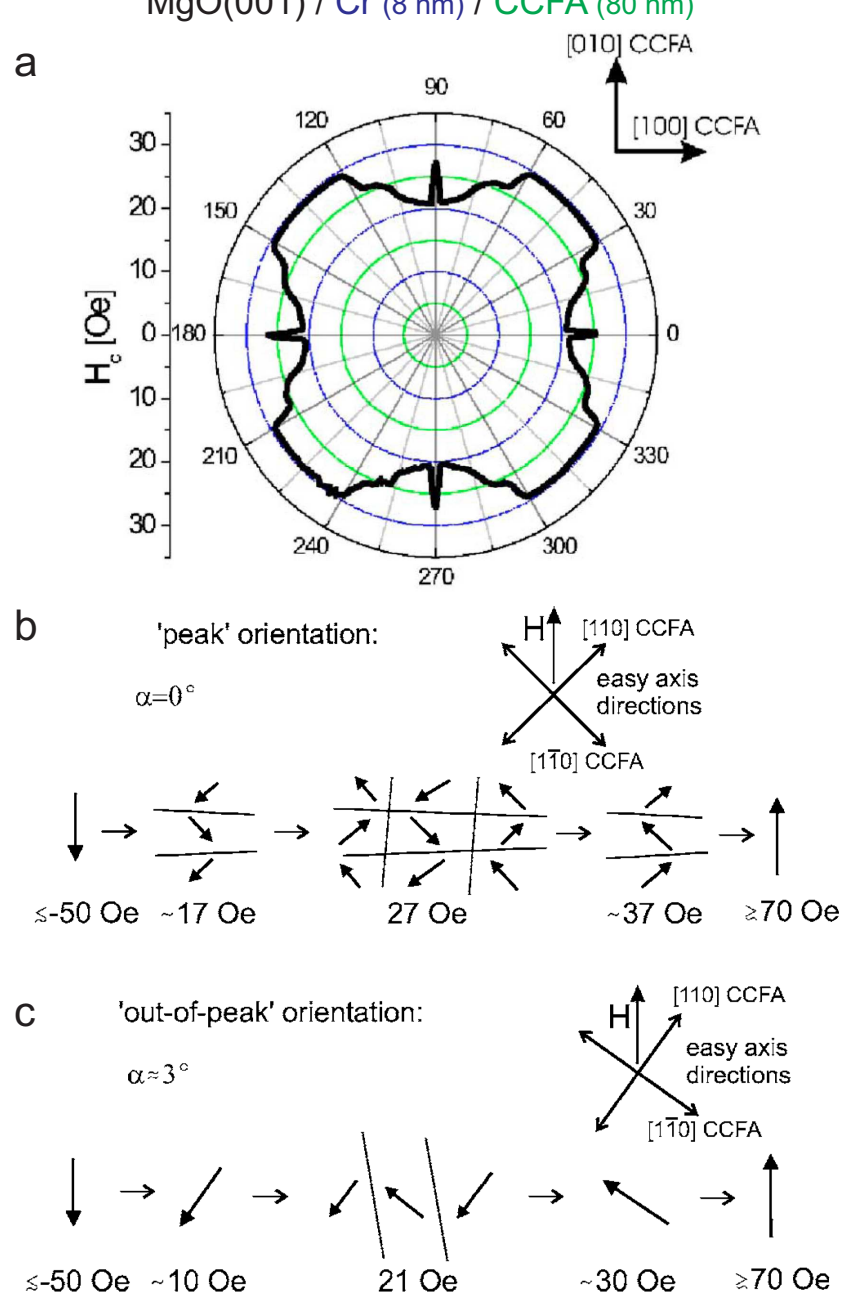

FIG. 6. (a) Polar plot of the coercive field $H_{\mathrm{c}}$ measured as a function of in-plane orientation for a $\mathrm{Co}_{2} \mathrm{Cr}_{0.6} \mathrm{Fe}_{0.4} \mathrm{Al}(001)$ thin film. CCFA $=\mathrm{Co}_{2} \mathrm{Cr}_{0.6} \mathrm{Fe}_{0.4} \mathrm{Al}$. Sketch of the magnetization reversal when $H$ is parallel to the $\langle 100\rangle \mathrm{Co}_{2} \mathrm{Cr}_{0.6} \mathrm{Fe}_{0.4} \mathrm{Al}$ direction (peak orientation) (b) and when $H$ is slightly deviated from the $\langle 100\rangle \mathrm{Co}_{2} \mathrm{Cr}_{0.6} \mathrm{Fe}_{0.4} \mathrm{Al}$ direction (out-of-peak orientation) (c). Reprinted with permission from [115]. Copyright 2006, American Institute of Physics. (Colour online)

origin of these peaks, MOKE microscopy [92] was used to study the magnetic domain configuration during magnetization reversal. The magnetization reversal process is schemed in Fig. 6b-c. When the magnetization reversal was examined along the [100] hard axes, stripes with $90^{\circ}$ domain walls appeared, due to magnetic frustration between two energetically equivalent easy axes (see Fig. 6b). In the demagnetized state $(H \approx 27 \mathrm{Oe})$, the stripes transformed into a checkerboard magnetic domain structure. In out-of-peak orientations (ca. $3^{\circ}$, Fig. 6c), magnetization reversal did not pass through this checker-board configuration, and a smaller coercivity was observed. As such, the origin of the peaks in the coercivity near the hard axes was attributed to the magnetization reversal process. 


\begin{tabular}{|c|c|c|c|}
\hline Compound & Method & $K$ & Reference \\
\hline \multirow[t]{2}{*}{$\mathrm{Co}_{2} \mathrm{Cr}_{0.6} \mathrm{Fe}_{0.4} \mathrm{Al}^{1)}$} & SQUID & $K_{1}=2.2 \mathrm{~kJ} / \mathrm{m}^{3} ; K_{\mathrm{u}}=3.7 \mathrm{~kJ} / \mathrm{m}^{3}$ & \\
\hline & & $K_{1}=1.1 \mathrm{~kJ} / \mathrm{m}^{3} ; K_{\mathrm{u}}=1.8 \mathrm{~kJ} / \mathrm{m}^{3}$ & {$[102,103]$} \\
\hline $\mathrm{Co}_{2} \mathrm{Cr}_{0.6} \mathrm{Fe}_{0.4} \mathrm{Al}$ & BLS & $K_{1}=2.0 \mathrm{~kJ} / \mathrm{m}^{3}$ & {$[104]$} \\
\hline $\mathrm{Co}_{2} \mathrm{MnAl}$ & TR-MOKE & $K_{1}=-0.4572 \mathrm{~kJ} / \mathrm{m}^{3}$ & {$[105]$} \\
\hline $\mathrm{Co}_{2} \mathrm{MnSi}^{2)}$ & BLS & $K_{1}=-1.0$ to $-9.0 \mathrm{~kJ} / \mathrm{m}^{3}$ & {$[62]$} \\
\hline \multirow{2}{*}{$\mathrm{Co}_{2} \mathrm{MnSi}^{2)}$} & FMR & $K_{1}=8.0$ to $-0.5 \mathrm{~kJ} / \mathrm{m}^{3}$ & \\
\hline & & $K_{\mathrm{u}}=0$ to $0.2 \mathrm{~kJ} / \mathrm{m}^{3}$ & {$[106]$} \\
\hline $\mathrm{Co}_{2} \mathrm{MnGe}$ & MOKE & $K_{1} / M_{\mathrm{s}}=-3.3 \mathrm{mT}, K_{\mathrm{u}} / M_{\mathrm{s}}=2.0 \mathrm{mT}$ & {$[107]$} \\
\hline \multirow[t]{2}{*}{$\mathrm{Co}_{2} \mathrm{MnGe}$} & FMR & $K_{1} / M_{\mathrm{s}}=-13.3 \mathrm{mT}$ & \\
\hline & & $K_{\mathrm{u}} / M_{\mathrm{s}}=3.5 \mathrm{mT}, K_{\perp} / M_{\mathrm{s}}=-120 \mathrm{mT}$ & {$[108,109]$} \\
\hline \multirow[t]{2}{*}{$\mathrm{Co}_{2} \mathrm{MnGe}^{3)}$} & FMR & $K_{\mathrm{u}} / M_{\mathrm{s}} \approx 0.25$ to $0.75 \mathrm{mT}$ & \\
\hline & & $K_{1} / M_{\mathrm{s}} \approx-0.50$ to $1.1 \mathrm{mT}$ & {$[110]$} \\
\hline \multirow[t]{2}{*}{$\mathrm{Co}_{x} \mathrm{Mn}_{y} \mathrm{Ge}_{1-x-y}{ }^{4)}$} & MOKE & $K_{\mathrm{u}} / M_{\mathrm{s}} \approx-2.0$ to $+4.0 \mathrm{mT}$ & \\
\hline & & $K_{6} / M_{\mathrm{s}} \approx 0.07$ to $0.15 \mathrm{mT}$ & {$[111,112]$} \\
\hline \multirow[t]{2}{*}{$\left.\mathrm{Co}_{2} \mathrm{FeSi}^{2}\right)$} & SQUID & $K_{\mathrm{u}} \approx 2.0$ to $20.0 \mathrm{~kJ} / \mathrm{m}^{3}$ & \\
\hline & & $K_{1} \approx 2.0$ to $4.0 \mathrm{~kJ} / \mathrm{m}^{3}$ & {$[113]$} \\
\hline $\mathrm{Co}_{2} \mathrm{FeSi}$ & SQUID & $K_{1}=1.8 \mathrm{~kJ} / \mathrm{m}^{3} ; K_{\mathrm{u}}=6.3 \mathrm{~kJ} / \mathrm{m}^{3}$ & {$[114]$} \\
\hline
\end{tabular}

TABLE III. Anisotropy constants for various $\mathrm{Co}_{2} M^{\prime} Z$ compound thin films

This magnetization reversal process is dependent on the sample structure, as when a $105 \mathrm{~nm}$ thick $\mathrm{Co}_{2} \mathrm{Cr}_{0.6} \mathrm{Fe}_{0.4} \mathrm{Al}$ film was grown on Fe-buffered $\mathrm{MgO}$, peaks in the coercive field were not observed at the hard axes [115]. Magnetization reversal occurred as depicted in Fig. 6c.

This type of magnetization reversal was also observed for $\mathrm{Co}_{2} \mathrm{MnSi}$ samples [62].

Case study 2: Multi-step magnetization reversal

Despite the expected four-fold anisotropy from the cubic Heusler materials, it is often observed that the anisotropy instead has a uniaxial character. In addition, it was widely observed that multi-step magnetization reversal occurs. For example, multi-step switching was noticed in

- $\mathrm{Co}_{2} \mathrm{MnGe}$ grown on a-plane $\mathrm{Al}_{2} \mathrm{O}_{3}$ [110] and $\operatorname{GaAs}(001)$ [107-109];

- $\mathrm{Co}_{2} \mathrm{MnSi}$ grown on $\operatorname{GaAs}(001)$ [116-118];

- $\mathrm{Co}_{2} \mathrm{FeSi}$ grown on $\mathrm{GaAs}(001)$ [113] ;

- $\mathrm{Co}_{2} \mathrm{MnGa}$ grown on $\mathrm{GaAs}(001)$ [119];

- $\mathrm{Co}_{2} \mathrm{Cr}_{0.6} \mathrm{Fe}_{0.4} \mathrm{Al}$ grown on $\operatorname{GaAs}(001)$ [102, 103, 120];

- $\mathrm{Co}_{2} \mathrm{MnSn}_{1-x} \mathrm{Sb}_{x}$ grown on $\operatorname{GaAs}(001)$ [121]; and

- $\mathrm{Co}_{2} \mathrm{Fe}_{0.5} \mathrm{Al}_{0.5}$ Si grown on $\mathrm{MgO}(001)$ [63].
As an example, in Fig. 7a-c we show magnetization $v s$ field loops measured by SQUID magnetometry for a $25 \mathrm{~nm}$ thick $\mathrm{Co}_{2} \mathrm{MnGe}(001)$ film, grown on $\mathrm{GaAs}(001)$ by molecular beam epitaxy, reported by Ambrose et al. [108]. When the field is applied along the [100] direction, a rounded loop characteristic of a hard axis is observed, see Fig. 7a. While in $\mathrm{Co}_{2} \mathrm{MnGe}$ the [110] and [110] directions are crystallographically equivalent, the comparison of Fig. 7b-c clearly shows these are not magnetically equivalent, even though they both show rectangular features associated to easy axes of direction. An in-plane study of the FMR resonance fields (Fig. $7 \mathrm{~d}, \theta$ is the angle between the applied field and the [100] hard axis) shows that two anisotropies are present. First, a fourfold cubic anisotropy, attributed to the magnetocrystalline anisotropy of $\mathrm{Co}_{2} \mathrm{MnGe}$, is evident. The inequivalence of the $\langle 110\rangle$ directions is again evidenced, as the resonance field differ for $\theta= \pm 45^{\circ}$, and is attributed to a uniaxial anisotropy, which is superimposed on the cubic anisotropy. Note that this uniaxial anisotropy is coincident with one of the $\langle 110\rangle$ directions.

Yang and coworkers [107] studied this multistep magnetic switching in more detail for $\mathrm{Co}_{2} \mathrm{MnGe}$ grown on $\operatorname{GaAs}(001)$. There the free energy density $\mathcal{E}$ was given as

$$
\mathcal{E}=K_{1} \alpha_{1}^{2} \alpha_{2}^{2}+K_{u} \sin ^{2} \theta-\mathbf{H} \cdot \mathbf{M}
$$

where $K_{1}$ and $K_{u}$ are the cubic and uniaxial anisotropy constants, $\alpha_{1}$ and $\alpha_{2}$ are the directional cosines with respect to the in-plane [100] and [010] axes $\left(\alpha_{1}=\theta+45^{\circ}\right.$ 

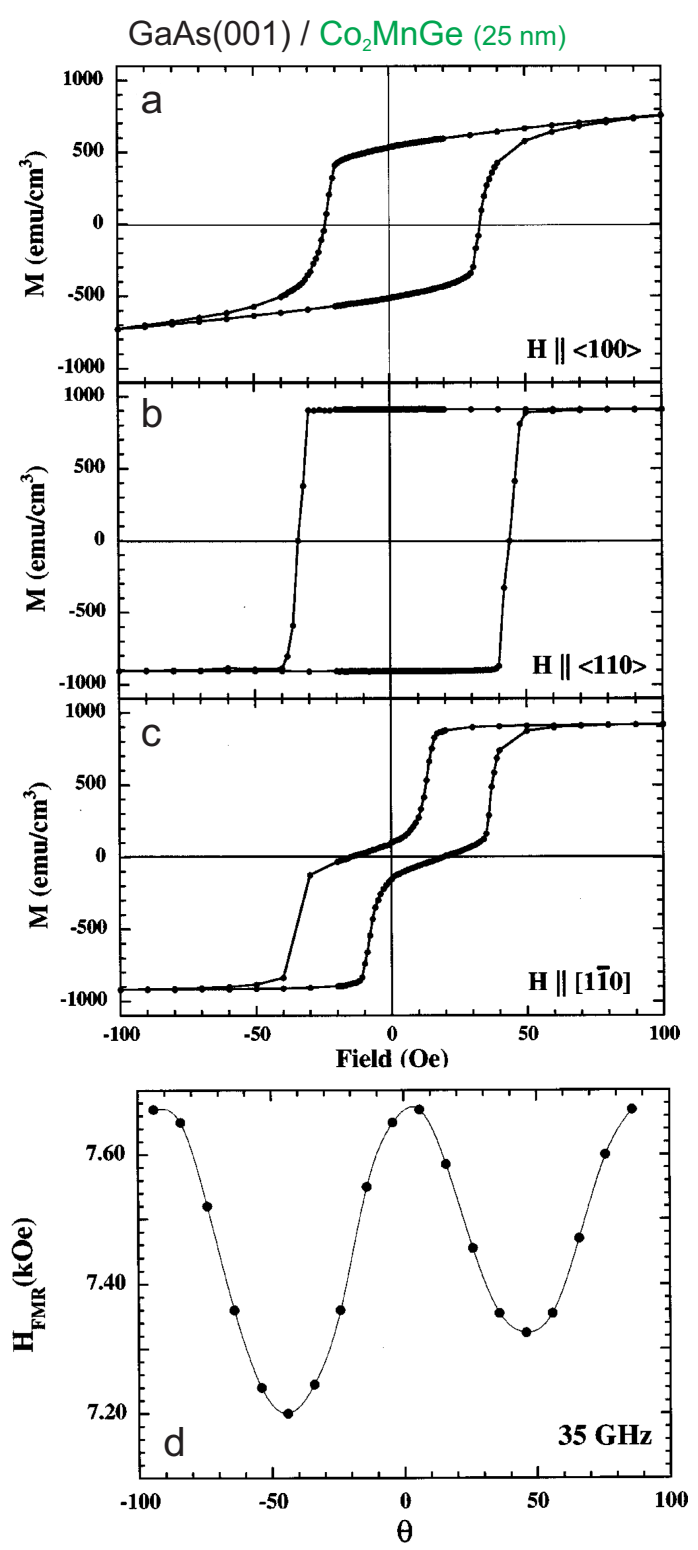

FIG. 7. Hysteresis curves measured at room temperature for a $250 \AA \mathrm{Co}_{2} \mathrm{MnGe}$ film with the magnetic field applied in the plane of the film along the (a) $\langle 100\rangle$ (b) [110] and (c) [110] directions. (d) Angular dependence of the FMR resonance field vs $\theta$ where $\theta$ is the angle between the applied magnetic field and the hard axis direction [100]. The solid line is a fit. Reprinted with permission from [108]. Copyright 2000, American Institute of Physics. (Colour online)

and $\left.\alpha_{2}=\theta-45^{\circ}\right), \theta$ is the angle between the magnetization $\mathbf{M}$ and the [110] axis, and the last term is the Zeeman energy. Two easy axes are present in this coordinate system, with the easiest axis along the [110] direction (coincident with the uniaxial anisotropy axis, i.e. $\left.\theta=0^{\circ}\right)$, and the second easy axis along the $[1 \overline{1} 0]$ direction $\left(\theta=90^{\circ}\right)$. The hard axes were estimated to lie at angles $\theta=63^{\circ}$ and $117^{\circ}$, tilted $27^{\circ}$ from the second easy axis. Using Eq. 8 and assuming coherent rotation of the magnetization, a very good agreement was found between the measured and simulated loops.

To confirm this model, the process of magnetization switching was investigated by Yang using the magnetooptical indicator film (MOIF) domain imaging technique [107]. The hysteresis loop, measured by vibrating sample magnetometry, with $H$ parallel to the second easy axis $\left(\theta=90^{\circ}\right)$ is shown at the bottom of Fig. 8 . The analysis of the MOIF domain images acquired at different points of the hysteresis loop are shown in Fig. 8a-i. Briefly, at high field $\mathbf{M}$ is aligned parallel to $\mathbf{H}$, along the second easy axis (Fig. 8a-b). Magnetization switching first involves the formation of $90^{\circ}$ domains (Fig. 8c), where $\mathbf{M}$ aligns along the easiest axis. This domain will then coherently rotate (Fig. 8d-f), until a new $90^{\circ}$ domain forms in the second switching step (Fig. 8g), again aligning $\mathbf{M}$ along the second easy axis (Fig. 8h-i). In the intermediate step (Fig. 8d-f), the magnetization is quasi-perpendicular to the applied field, and as such a near-zero magnetization is measured between the two switching events.

This model, where cubic and uniaxial anisotropies are superimposed and coincident [108, 109], was later invoked by several groups to rationalize similar results for Heusler compounds epitaxially grown on $\mathrm{GaAs}(001)$ substrates [102, 103, 107, 117, 118].

While we have assigned the cubic anisotropy to magnetocrystalline energy, we have up to now neglected to describe the origin of the uniaxial anisotropy. To this day, the origin of this uniaxial anisotropy is not clearly understood. Several explanations have been put forward, as mentioned in reports by Ambrose [108, 109], Uemura [102] and Wang [117], including: surface reconstruction of the GaAs substrate, the formation of a surface alloy, or an anisotropy of the dangling covalent bonds at the surface of $\operatorname{GaAs}(001)$. This latter explanation [122] is particularly attractive, as the dangling bonds of either Ga- or As-terminated (001) surfaces are aligned with the $\langle 110\rangle$ in-plane directions, as is the observed uniaxial anisotropy. For $\mathrm{Co}_{2} \mathrm{MnSi}$ thin films grown on $\mathrm{GaAs}(001)$, Singh and coworkers report that this uniaxial asymmetry dominated, as they observed a very clear two-fold symmetry in polar plots of the coercive field, where the easy axis was parallel to $\langle 110\rangle[123]$. It is also worth mentioning that under certain deposition conditions, Singh observed an interface reaction leading to the formation of $\mathrm{Mn}_{2} \mathrm{As}$.

However, the occurrence of this uniaxial anisotropy is not as easily understood when Heusler films are grown on the cubic $\mathrm{MgO}$ substrate. For example, the uniaxial anisotropy was almost unaffected whether $\mathrm{Co}_{2} \mathrm{Cr}_{0.6} \mathrm{Fe}_{0.4} \mathrm{Al}$ was grown on GaAs with or without a $1.5 \mathrm{~nm} \mathrm{MgO}$ buffer $[102,103]$. However, if the $\mathrm{MgO}$ buffer thickness was increased to $3.0 \mathrm{~nm}$ for $\mathrm{Co}_{2} \mathrm{MnSi}$ on GaAs, the anisotropy was markedly decreased, though still present. However, using a $\mathrm{MgO}$-buffered $\mathrm{MgO}$ substrate does not eliminate this particular anisotropy, as similar (albeit less pronounced) magnetization switching features were observed for $\mathrm{Co}_{2} \mathrm{Fe}_{0.5} \mathrm{Al}_{0.5} \mathrm{Si}$ grown on 


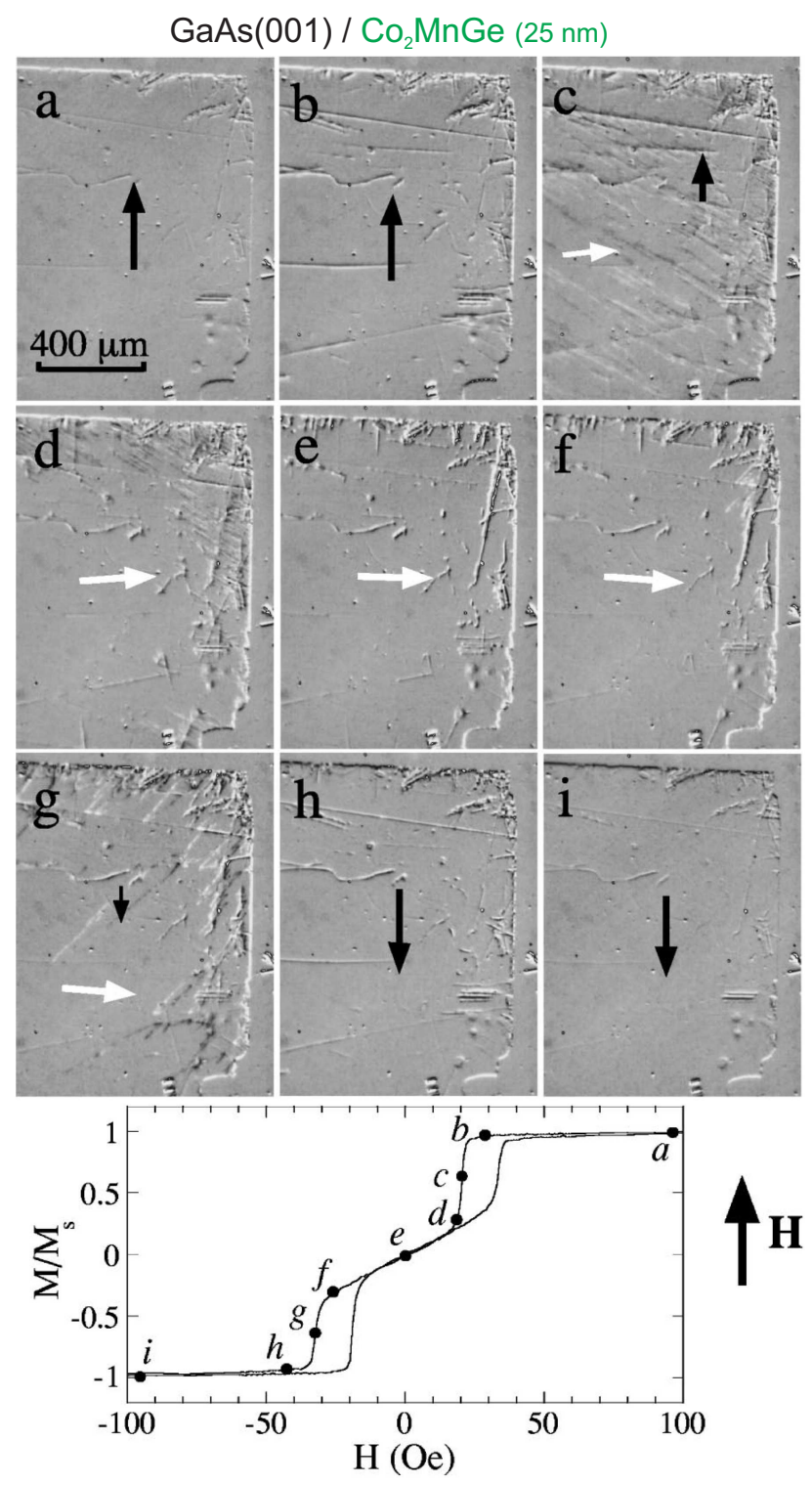

FIG. 8. The magneto-optical indicator film images of the $\mathrm{Co}_{2} \mathrm{MnGe}(001)$ film with $H$ parallel to the second-easy axis $\left(\theta=90^{\circ}\right)$. The easiest axis is horizontal. The nine points marked in the hysteresis loop in the lower part indicate where image (a) to (i) were taken: (a) $H=100 \mathrm{Oe}$, (b) $H=29$ Oe, (c) $H=20 \mathrm{Oe},(\mathrm{d}) H=18 \mathrm{Oe},(\mathrm{e}) H=0 \mathrm{Oe},(\mathrm{f}) H=-27 \mathrm{Oe}$, (g) $H=-31$ Oe, (h) $H=-42$ Oe, (i) $H=-100$ Oe. The direction of the applied magnetic field is illustrated on the right of the hysteresis loop. Reprinted figure with permission from Yang, Shang, Chien, Ambrose, Krebs, Prinz, Nikitenko, Gornakov, Shapiro, Shull, Phys. Rev. B 65174410 (2002) [107]. Copyright (2002) by the American Physical Society. (Colour online)

$\mathrm{MgO}(001)$ [63]. In this last study, Wang et al. proposed the uniaxial anisotropy may be due to the lattice misfit of $-4.5 \%$ between $\mathrm{MgO}$ and $\mathrm{Co}_{2} \mathrm{Fe}_{0.5} \mathrm{Al}_{0.5} \mathrm{Si}$.

Case study 3: Quadratic MOKE

In MOKE magnetometry, one usually only observes
$\mathrm{MgO}(001) / \mathrm{Cr}(40 \mathrm{~nm}) / \mathrm{Co}_{2} \mathrm{MnSi}(30 \mathrm{~nm})$

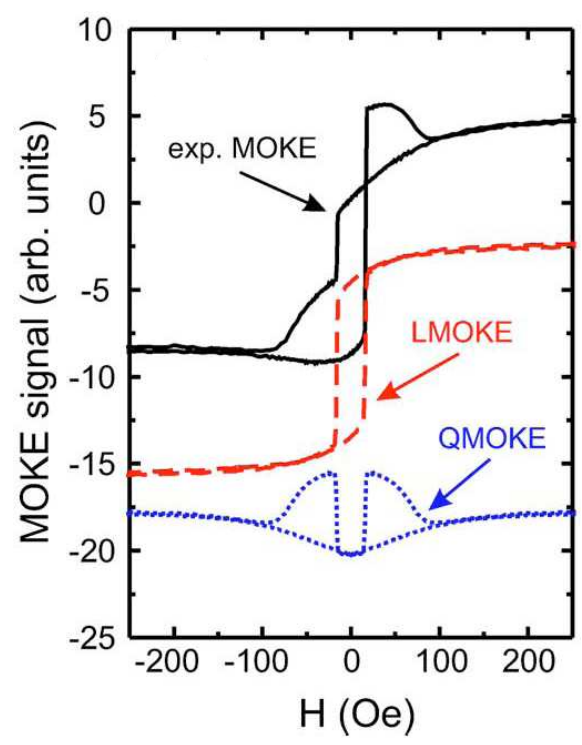

FIG. 9. Room temperature hysteresis loops measured by MOKE magnetometry for a $\mathrm{Co}_{2} \mathrm{MnSi}$ film (full line). Symmetrization and antisymmetrization of these loops provide the LMOKE (dashed line) and QMOKE (dotted line) contributions. Reprinted with permission from [62]. Copyright 2008, American Institute of Physics. (Colour online)

the magneto-optical response that is proportional to the longitudinal magnetization $M_{\mathrm{L}}$ of the sample, the LMOKE. Considering the dielectric tensor $\epsilon$ of a material, this is equivalent to observing only terms that are directly proportional to the magnetization [124],

$$
\epsilon_{i j}=\epsilon_{i j}^{(0)}+K_{i j k} M_{k}
$$

where

$$
K_{i j k}=\frac{\partial \epsilon_{i j}}{\partial M_{k}}
$$

However, there have been several reports of MOKE studies where the observed Kerr rotation hysteresis loops are no longer symmetric upon field inversion, but also contain an even component, as is shown in Fig. 9. In this figure, the two components were obtained by an arithmetic symmetrization and asymmetrization procedure [62]. This effect is understood when considering the magneto-optical response of the dielectric tensor, taking into account terms that are proportional to second order magnetization terms, the quadratic MOKE (QMOKE) [124],

$$
\epsilon_{i j}=\epsilon_{i j}^{(0)}+K_{i j k} M_{k}+G_{i j k l} M_{k} M_{l},
$$

where

$$
G_{i j k l}=\frac{\partial^{2} \epsilon_{i j}}{\partial M_{k} \partial M_{l}}
$$




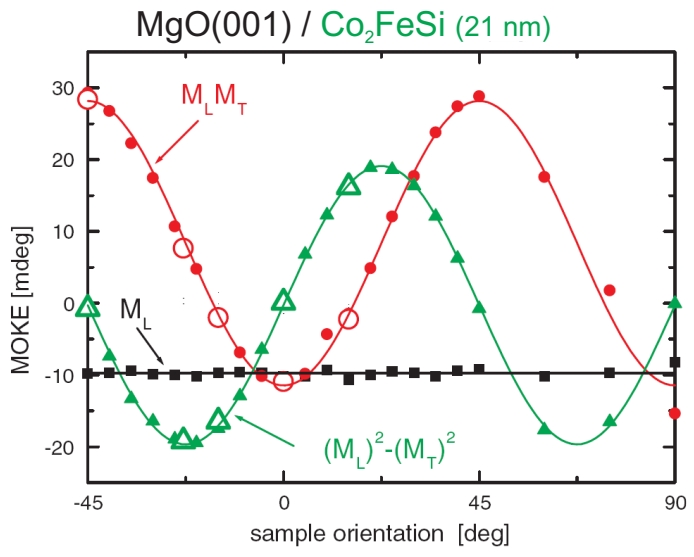

FIG. 10. Dependence of different MOKE signals in saturation for a $21 \mathrm{~nm}$ thick $\mathrm{Co}_{2} \mathrm{FeSi}(100)$ film as a function of inplane orientation which are determined from the 8-directional method (full symbols) or from the height of peaks in QMOKE loops (open symbols). Reprinted with permission from [127]. Copyright 2007, Institute of Physics. (Colour online)

The expected Kerr rotation for a cubic material, when taking into account effects up to the second order of the magnetization [125], is the real part of

$$
\begin{aligned}
& \Phi_{\mathrm{s}}=\mathcal{A} \cdot K M_{\mathrm{L}}+\mathcal{B}\left[\left(-\frac{K^{2}}{\tilde{n}^{2}}+2 G_{44}+\frac{\Delta G}{2}\right) M_{\mathrm{L}} M_{\mathrm{T}}\right. \\
& \left.-\frac{1}{2} \Delta G \cos 4 \alpha \cdot M_{\mathrm{L}} M_{\mathrm{T}}-\frac{1}{4} \Delta G \sin 4 \alpha\left(M_{\mathrm{L}}^{2}-M_{\mathrm{T}}^{2}\right)\right]
\end{aligned}
$$

where $\mathcal{A}$ and $\mathcal{B}$ are (complex) optical weighting factors [126], $K=K_{i j k}$ and $G_{44}=G_{1212}=G_{1313}=G_{2323}$ are components of the linear and quadratic magneto-optical tensors, respectively, $\Delta G=G_{11}-G_{12}-2 G_{44}$ (where $G_{11}=G_{i i i i}, G_{12}=G_{i i j j}$ ) embodies the magneto-optical anisotropy, and $\tilde{n}$ is the complex refractive index of the material.

The amplitudes of the components of the Kerr rotation proportional to $M_{\mathrm{L}}, M_{\mathrm{L}} M_{\mathrm{T}}$, and $M_{\mathrm{L}}^{2}-M_{\mathrm{T}}^{2}$ in saturation can be determined by measuring the Kerr rotation at perpendicular light incidence onto the sample for eight different applied field directions for each in-plane sample orientation. These angles are chosen such that either $M_{\mathrm{L}}$ or $M_{\mathrm{T}}$ is equal to zero (such that the $M_{\mathrm{L}} M_{\mathrm{T}}$ contribution in Eq. 13 is equal to zero), or $M_{\mathrm{L}}=M_{\mathrm{T}}$ (in which case $\left.M_{\mathrm{L}}^{2}-M_{\mathrm{T}}^{2}=0\right)$. Such a series of measurements is shown in Fig. 10 for a $21 \mathrm{~nm}$ thick $\mathrm{Co}_{2} \mathrm{FeSi}$ thin film. The amplitude of the QMOKE observed for this sample, $18 \mathrm{mdeg}$, is the highest ever reported for any material [127].

In addition to $\mathrm{Co}_{2} \mathrm{FeSi}[127,128]$, this effect has been noticed in $\mathrm{Co}_{2} \mathrm{MnSn}_{1-x} \mathrm{Sb}_{x}$ [121], $\mathrm{Co}_{2} \mathrm{MnSi}[62,129]$ and $\mathrm{Co}_{2} \mathrm{MnGe}[111,112,130]$. However, the amplitude of the QMOKE signal is not as strong (2 mdeg and 0.4 mdeg for $\mathrm{Co}_{2} \mathrm{MnSi}$ [129] and $\mathrm{Co}_{2} \mathrm{MnGe}$ [130], respectively) as in $\mathrm{Co}_{2} \mathrm{FeSi}$. It is worth noting this effect is not restricted to Heusler compounds, but has also been seen in (amongst other systems) NiFe bilayers [131, 132] and thin Fe films [125, 133-135]. The microscopic origin of this large effect in Heusler compounds is not yet clearly understood, but is believed to be related to second order spin-orbit coupling [136]. To aid in the systematic study of this effect, Trudel and coworkers have recently built a dedicated dual-beam MOKE system equipped with a quadrupole magnet, enabling an automated evaluation of the QMOKE in saturation [129].

It is worth noting that while the angular dependence of the magnetization reversal (as seen in polar plots of the coercivity) might strongly deviate from that expected for a material with cubic symmetry, the QMOKE still follows the expected behaviour of Eq. 13. This is because these two methods look at the sample in a different magnetization state: the coercivity characterizes the demagnetized state, while the QMOKE measurements represent the saturated state. As such, the two analyses are not contradictory, but rather complementary [130].

Case study 4: Effect of annealing $\mathrm{Co}_{2} \mathrm{MnSi}$ on its anisotropy

A method conducive to the quantitative study of magnetic anisotropy is Brillouin light scattering (BLS) spectroscopy (see Eq. 14.10 in ref. [100] and accompanying discussion). Briefly, BLS spectra are collected for many in-plane orientations with respect to the applied magnetic field, which is sufficiently strong to magnetically saturate the sample. The observed spin-wave frequencies show a periodic variation as a function of in-plane orientation. Upon fitting, the anisotropy constant can be determined [100].

Gaier et al. have used this method to study the effect of annealing on a series of $\mathrm{Co}_{2} \mathrm{MnSi}$ thin films [62]. X-ray diffraction showed that upon annealing, $\mathrm{L} 2{ }_{1}$ ordering increases. The frequency of the Damon-Esbach mode as a function of in-plane orientation is shown in Fig. 11a for a $\mathrm{Co}_{2} \mathrm{MnSi}$ film annealed at $375^{\circ} \mathrm{C}$. As can be seen, the spin wave frequency reaches a minimum for the in-plane $\langle 100\rangle$ directions, and a maximum for the $\langle 110\rangle$ directions. The maximum identifies the easy direction, where the magnetization is oriented along the applied magnetic field, maximizing the effective field, resulting in a higher spin wave frequency [100]. Also shown in Fig. 11a is the best fit to the measured spin wave frequencies, quantifying the cubic anisotropy constant $K_{1}$. This procedure was repeated for various annealing temperatures, which provided different degrees of $\mathrm{L} 2{ }_{1}$ ordering. It was observed that this increase in L $2{ }_{1}$ ordering is accompanied by an initial increase of the cubic anisotropy constant $K_{1}$, reaching a maximum for $375^{\circ} \mathrm{C}$, and then a decrease by an order of magnitude, see Fig. 11b. When considering Eqs. 6-7, this change may be associated with a decrease in the spinorbit coupling parameter $\xi(r)$, or the anisotropy of the angular momentum $\mathbf{L}$. The latter explanation is more likely, as even though the local environment and its associated ligand fields may affect $\xi(r)$, this parameter is predominantly an atomic property [93]. 

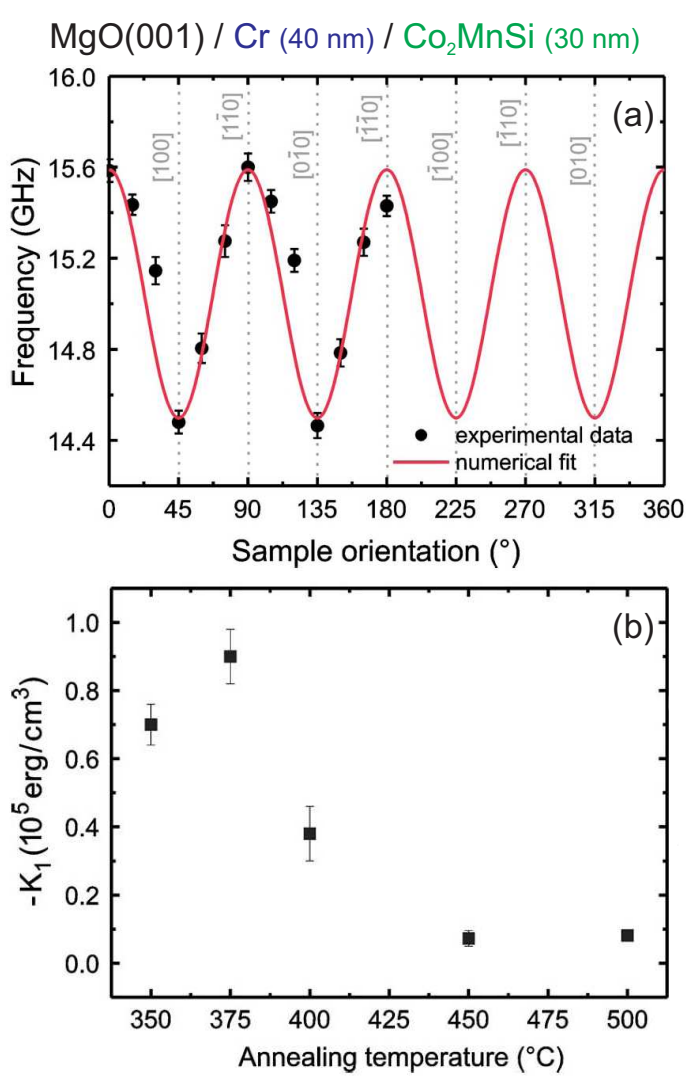

FIG. 11. (a) Frequency of the Damon-Esbach mode as a function of the angle between the external magnetic field $H=300$ Oe and the [110] easy axis direction for the $\mathrm{Co}_{2} \mathrm{MnSi}$ film annealed at $375^{\circ} \mathrm{C}$. (b) Cubic volume anisotropy constant $K_{1}$ for $\mathrm{Co}_{2} \mathrm{MnSi}$ films annealed at different temperatures. Reprinted with permission from [62]. Copyright 2008, American Institute of Physics. (Colour online)

\section{EXCHANGE CONSTANT AND SPIN-WAVE STIFFNESS}

Using the simple Jullière model [137], the tunnelling magnetoresistance (TMR) ratio of a ferromagnet/insulator/ferromagnet tunneling magnetic junction is expressed as (under the conditions of incoherent tunnelling, such as with an amorphous aluminum oxide barrier)

$$
T M R=\frac{2 P_{1} \cdot P_{2}}{1-P_{1} \cdot P_{2}}
$$

where $P$ stands for the spin polarization (c.f. Eq. 1) of the two ferromagnetic electrodes in the MTJ. High spin polarizations therefore lead to high TMR ratios. As such, half-metallic Heusler compounds, with their $100 \%$ spin polarization, are ideal contenders towards high-performance spintronic devices.

While high TMR ratios have indeed been observed in Heusler-based MTJs, indicating a high spin polarization at low temperature, a commonly observed feature is the decay of TMR ratios with increasing temperature. Equa- tion 14 intuitively identifies the decay of the spin polarization as the origin of the strong temperature dependence of the TMR ratios.

For Heusler compounds to be integrated in mainstream devices, there is a pressing need to understand the origin of this reduction of the idealized $100 \%$ spin polarization at finite temperature [16, 21, 138]. Proposed mechanisms for this phenomenon include inelastic electron-magnon interactions, creating states at the Fermi level in the minority spin channel band gap $[23,138]$, and the thermal excitation of magnons which decrease the spin polarization [16]. Furthermore, in the case of coherent tunneling (such as through an $\mathrm{MgO}$ barrier), the presence of magnons decreases the TMR ratio due to the increased tunneling propability of minority spins in the antiparallel configuration [139, 140]. For all of these mechanisms, the creation of magnons (spinwaves) is at the center of the problem.

As such, the investigation of magnons, and hence the exchange interaction, is an important issue in order to understand the strong temperature dependence of the spin polarization in Heusler compounds. Knowledge of the exchange strength is also crucial for micromagnetic simulations and the study of dynamic phenomena.

Different expressions for the exchange strength can be found through the literature. The most common expressions are the spin wave stiffness $D$, the exchange constant $A$, and the Heisenberg exchange integral $J$. This latter expression is particularly encountered in theoretical descriptions of the exchange.

The Heisenberg Hamiltonian uses the exchange integral $J$ to describe the energy between pairs of spins [93],

$$
\mathcal{H}_{\text {Heisenberg }}=-\sum_{j \neq i} J_{i j} \mathbf{S}_{\mathbf{i}} \cdot \mathbf{S}_{\mathbf{j}}
$$

The energy of an exchange-dominated spin wave with wavevector $\mathbf{q}$ is well described in terms of the exchange integral $J_{i j}$ through a Fourier transformation [141]

$$
E(\mathbf{q})=\frac{4 \mu_{\mathrm{B}}}{\mu} \sum_{j \neq 0} J_{0 j}\left(1-\exp \left[i \mathbf{q} \cdot \mathbf{R}_{0 j}\right]\right)
$$

where $\mathbf{R}_{0 j}=\mathbf{R}_{0}-\mathbf{R}_{j}$ is a real-space lattice vector, and here $\mu$ is the moment per atom in units of $\mu_{\mathrm{B}}$. When one only considers nearest-neighbour interactions in a onedimensional case (i.e. $\mathbf{R}_{0 j}=a$ where $a$ is the lattice parameter and $\left.J_{0 j}=J\right)$, one gets the simple result that the energy $E$ of a spin wave is

$$
E=\hbar \omega=\left(J S a^{2}\right) q^{2}=D q^{2},
$$

which is also valid for the three cubic lattices [142]. The spin wave stiffness $D$ describes the energy of a spin wave (neglecting dipolar interaction), and represents the parabolic curvature in the magnon dispersion at the dispersion minimum for $q=0$ (i.e. at the $\Gamma$-point) - see $e . g$. Fig. 7 in [89]. 
Finally, the spin wave stiffness $D$ and the exchange constant $A$ are related by the simple expression [143]

$$
A=\frac{D M_{\mathrm{s}}}{2 g \mu_{\mathrm{B}}} .
$$

The exchange constant $A$ describes the exchange energy density $\mathcal{E}$ in a continuous magnetization model, $\mathcal{E}=A|\nabla \vec{m}|^{2}$. Such an expression is usually used for micromagnetic calculations, or the description of domain walls, where a continuous magnetization model is employed.

As $A$ and $D$ are accessible by temperature-dependent magnetization measurements [144], Brillouin light scattering spectroscopy [100] and neutron diffraction [142], they are natural exchange expressions bridging theory and experiment.

\section{A. Experimental determination of exchange}

The saturation magnetization of ferromagnetic materials is known to often decay with increasing temperature, due to the thermal excitation of spin waves, as

$$
M_{\mathrm{S}}(T)=M_{\mathrm{s}}(0 \mathrm{~K})\left(1-\mathcal{B} T^{3 / 2}\right),
$$

a behaviour known as Bloch's law [142, 145], with

$$
\mathcal{B}=2.612 \frac{V_{\mathrm{o}}}{\langle S\rangle}\left(\frac{k_{\mathrm{B}}}{4 \pi D}\right)^{3 / 2}
$$

where $V_{\mathrm{o}}$ is the atomic volume $\left(a^{3} / 16\right.$ for a full-Heusler compound), $\langle S\rangle=M_{\mathrm{s}} /\left(8 \mu_{\mathrm{B}}\right)$ is the average spin per atom (using $M_{\mathrm{S}}$ in $\mu_{\mathrm{B}} / \mathrm{fu}$ ), and $k_{\mathrm{B}}$ is Boltzmann's constant [144]. Thus, measuring the temperature dependence of the saturation magnetization by means of SQUID or vibrating sample magnetometry provides a means to determine the spin wave stiffness $D$. For example, Ritchie and coworkers used this method to determine $D=4.66 \mathrm{meV} \mathrm{nm}^{2}$ for bulk $\mathrm{Co}_{2} \mathrm{MnSi}$ [144].

For thin films, BLS spectroscopy is used to determine the spin wave stiffness. In the Damon-Esbach geometry (i.e. when the transferred wavector $\mathbf{q} \perp \mathbf{M}$, see Fig. 12), two types of spin waves are typically observed. Damon-Esbach modes are dominated by dipolar interactions, while perpendicular standing spin waves (PSSW) are exchange-dominated. PSSW modes are standing waves bound at the two thin film interfaces, and their wavevenumber $q_{n}$ is quantized by the film thickness $d$ such that $q_{n}=n \frac{\pi}{d}$. The observed spin wave frequency for PSSW modes can be approximated as (in SI units)

$$
\omega_{\mathrm{PSSW}}=\gamma \mu_{0} \sqrt{\left(H+\frac{2 A}{\mu_{0} M_{\mathrm{s}}} q_{n}^{2}\right)\left(H+M_{\mathrm{S}}+\frac{2 A}{\mu_{0} M_{\mathrm{S}}} q_{n}^{2}\right)},
$$

where $\gamma=\frac{1}{g_{\mathrm{e}}}\left|\gamma_{\mathrm{e}}\right| g$ is the gyromagnetic ratio $\left(\gamma_{\mathrm{e}}\right.$ is the gyromagnetic ratio of a free electron and $g_{\mathrm{e}}$ and $g$ are and

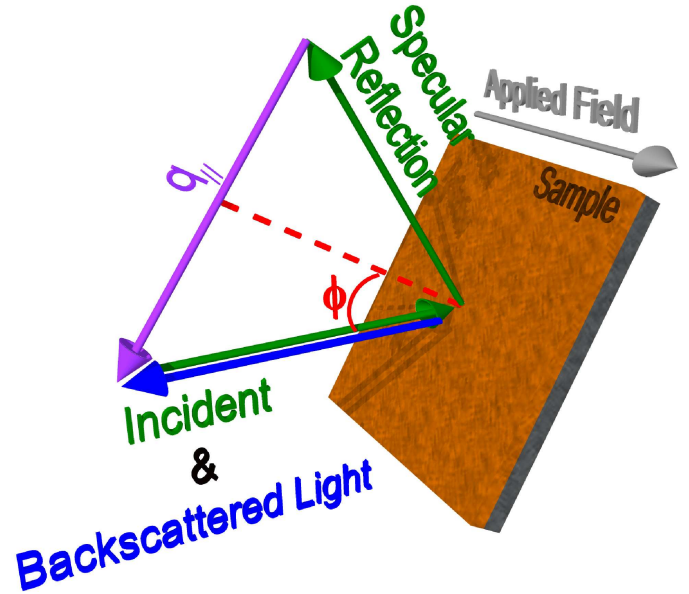

FIG. 12. Damon-Esbach geometry of BLS measurements. (Colour online)

the Landé factors for the free electron and the material, respectively), $\mu_{0}$ is the permeability of vacuum, and $A$ is the exchange constant.

To determine the exchange constant, one typically measures the spin wave frequencies at various applied fields, angles of incidence, and (if magneto-crystalline anisotropy is important) in-plane sample orientations. Equation 21 is usually too simple, as it neglects effects such as spin-wave mode crossings. Typically, spin wave frequency calculations are done using a model, such as the one based on a continuum-type magnetostatic theory $[146]$.

An example is provided in Fig. 13 where a $80 \mathrm{~nm}$ thick $\mathrm{Co}_{2} \mathrm{Cr}_{0.6} \mathrm{Fe}_{0.4} \mathrm{Al}$ thin film was studied by BLS spectroscopy [104]. Here the measured spin wave frequencies are plotted as a function of applied field strength along two high-symmetry directions (Fig. 13(a-b)), and as a function of in-plane orientation for a given field strength (Fig. 13c). The best fit yielded $A=4.8 \mathrm{pJ} / \mathrm{m}$, cubic volume anisotropy $K_{1}=2 \times 10^{3} \mathrm{~J} / \mathrm{m}^{3}$, Landé $g$-factor $=1.9$, and $M_{\mathrm{s}}=520 \mathrm{kA} / \mathrm{m}$. This corresponds to a spin wave stiffness $D$ of $2.03 \mathrm{meV} \mathrm{nm}^{2}$.

A compilation of exchange constants and spin wave stiffnesses determined by BLS spectroscopy is presented in Table IV. Accompanying this data are the corresponding $T_{\mathrm{c}}$ for the bulk Heusler compounds. As one might expect [90], the Curie temperature increases along with the exchange constant (see Eq. 5 above and related discussion).

In the same study that looked into the influence of $\mathrm{L} 2{ }_{1}$ ordering on the magneto-crystalline anisotropy (c.f. Fig. 11), Gaier et al. also evaluated the exchange constant $A$ for $\mathrm{CO}_{2} \mathrm{MnSi}$ samples grown on Cr-buffered $\mathrm{MgO}$ annealed at various temperatures [62]. For thermal treatment at increasing temperatures (and therefore increasing L $2{ }_{1}$ ordering), the exchange constant was seen to increase by $\sim 9 \%$. This is quite different than what was observed for the cubic anisotropy constant, which over the 

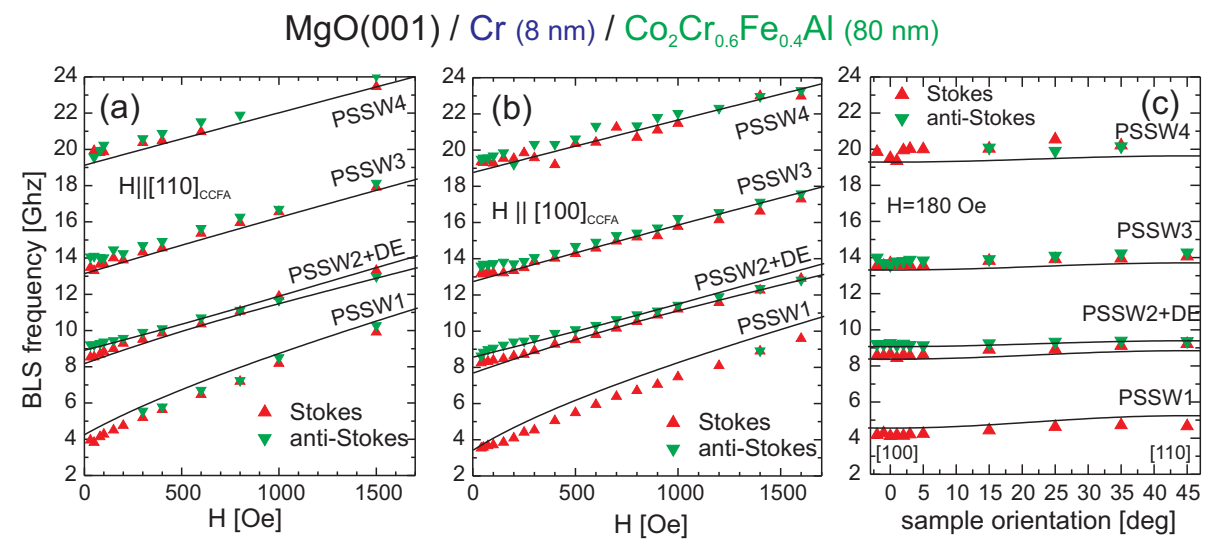

FIG. 13. Measured spin wave frequencies as a function of applied magnetic field $H \|[110]_{\mathrm{CCFA}}$ (a) and $H \quad \| \quad[100]_{\mathrm{CCFA}}$ (b). (c) Spin wave BLS frequencies as a function of sample orientation (the angle between [100] $]_{\text {CCFA }}$ and $H$. In (ac), solid lines are the calculated spin-wave frequencies. $\mathrm{CCFA}=\mathrm{Co}_{2} \mathrm{Cr}_{0.6} \mathrm{Fe}_{0.4} \mathrm{Al}$. Reprinted with permission from [104]. Copyright 2009, Institute of Physics. (Colour online)

\begin{tabular}{ccccccc}
\hline \hline \multirow{2}{*}{ Compound } & $\begin{array}{c}N_{\mathrm{v}} \\
{\left[\mathrm{e}^{-} / \text {f.u. }\right]}\end{array}$ & $\begin{array}{c}T_{\mathrm{C}}^{\text {bulk }} \\
{[\mathrm{K}]}\end{array}$ & $\begin{array}{c}\text { thin film } \\
\text { order }\end{array}$ & $\begin{array}{c}M_{\mathrm{s}} \\
{\left[\mu_{\mathrm{B}} / \mathrm{f} . \mathrm{u} .\right]}\end{array}$ & $\begin{array}{c}D \\
{\left[\mathrm{meV} \mathrm{nm}{ }^{2}\right]}\end{array}$ & $\begin{array}{c}A \\
{[\mathrm{pJ} / \mathrm{m}]}\end{array}$ \\
\hline \hline $\mathrm{Co}_{2} \mathrm{FeSi}^{1}$ & 30 & 1100 & $\mathrm{~L} 2{ }_{1}$ & 4.93 & $7.2(2)$ & $31.5(5)$ \\
$\mathrm{Co}_{2} \mathrm{MnSi}^{2}$ & 29 & 985 & $\mathrm{~L} 2{ }_{1}$ & 4.73 & $5.8(2)$ & $23.5(1)$ \\
$\mathrm{Co}_{2} \mathrm{MnGe}^{3}$ & 29 & 905 & - & 3.79 & $3.8(4)$ & $12.0(1.6)$ \\
$\mathrm{Co}_{2} \mathrm{Mn}_{0.77} \mathrm{Ge}_{0.42}$ & - & 905 & $\mathrm{~L} 22_{1}$ & 6.43 & $4.13(15)$ & $22.5(1.0)$ \\
$\mathrm{Co}_{2} \mathrm{FeAl}^{5}$ & 29 & $\approx 1000$ & $\mathrm{~B} 2$ & 5.21 & $3.7(1)$ & $15.5(5)$ \\
$\mathrm{Co}_{2} \mathrm{MnAl}^{6}$ & 28 & 693 & $\mathrm{~B} 2$ & 2.88 & 1.9 & 4.8 \\
$\mathrm{Co}_{2} \mathrm{Cr}_{0.6} \mathrm{Fe}_{0.4} \mathrm{Al}^{7}$ & 27.8 & 750 & $\mathrm{~B} 2$ & 2.65 & $2.03(16)$ & $4.8(4)$ \\
\hline${ }^{1}[10,147] ;{ }^{2}[45,60,143] ;{ }^{3}[45,110] ;{ }^{4}[45,130] ;{ }^{5}[81,104,148],{ }^{6}[45,81,149] ;{ }^{7}[80,104]$
\end{tabular}

TABLE IV. Spin wave stiffness and exchange constant of $\mathrm{Co}_{2}$-based Heusler compounds.

same sample series decreased by an order of magnitude. It is interesting to notice that spin-spin and spin-orbit coupling seem to react in different ways to the change of ordering.

We will take this opportunity to bring the reader's attention to the interpretation of such results. One has to be aware that $\mathrm{Cr}$ from the buffer layer may diffuse into the $\mathrm{Co}_{2} M^{\prime} Z$ compound upon annealing, thereby also altering the observed magnetic properties. For example, Tezuka [150] and Wang [63] have compared the magnetic properties of $\mathrm{Co}_{2} \mathrm{Fe}_{0.5} \mathrm{Al}_{0.5}$ Si films grown on $\mathrm{MgO}$ with and without a $\mathrm{Cr}$ buffer. In both cases, a sharp decrease in $M_{\mathrm{s}}$ and increase in $H_{\mathrm{c}}$ were observed above a threshold annealing temperature for Cr-buffered samples. These abrupt changes in $M_{\mathrm{s}}$ and $H_{\mathrm{c}}$ were not present for $\mathrm{MgO} / \mathrm{Co}_{2} \mathrm{Fe}_{0.5} \mathrm{Al}_{0.5} \mathrm{Si}$ structures, even up to an annealing temperature of $700^{\circ} \mathrm{C}$ [63]. As such, Cr interdiffusion was proposed as the origin of this discrepancy. In the study by Gaier [62], the maximum annealing temperature was $500^{\circ} \mathrm{C}$, which is below the threshold temperature observed by Wang [63]. The decrease in coercive field observed by Gaier would also be inconsistent with the results of Tezuka [150] and Wang [63], and as such the reported behaviour is likely to be inherent to the ordering degree of $\mathrm{Co}_{2} \mathrm{MnSi}$.

\section{B. Trends in exchange amongst the Heusler compounds}

As was discussed in Section II of this Review, the Heusler compounds are well known to be systematized by the number of valence electrons $N_{\mathrm{v}}$. Striking examples are the magnetic moment described by the Slater-Pauling rule $[39,83,87]$ or the Curie temperature $T_{C}[90]$, which are both generally proportional to $N_{\mathrm{v}}$. Here we discuss how the spin wave stiffness $D$ in Heusler compounds also appears to scale with $N_{\mathrm{v}}$ as well.

We have collected $D$ values of various $\mathrm{Co}_{2}$-based Heusler compounds. Most of these were determined by the current authors, namely: $\mathrm{Co}_{2} \mathrm{FeSi}\left(\mathrm{L} 2_{1}\right)$ [147], $\quad \mathrm{Co}_{2} \mathrm{MnSi}\left(\mathrm{L}_{1}\right) \quad$ [143], $\quad \mathrm{Co}_{2} \mathrm{FeAl}(\mathrm{B} 2) \quad$ [104], $\mathrm{Co}_{2} \mathrm{Cr}_{0.6} \mathrm{Fe}_{0.4} \mathrm{Al}(\mathrm{B} 2)$ [104], $\mathrm{Co}_{2} \mathrm{MnAl}(\mathrm{B} 2)$ [149], and $\left.\mathrm{Co}_{2} \mathrm{Mn}_{0.77} \mathrm{Ge}_{0.42}(\mathrm{~L} 2)_{1}\right)$ [130]. Belmeguenai et al. recently reported on the spin wave stiffness of $\mathrm{Co}_{2} \mathrm{MnGe}$ [110]. However, the crystallographic order of the investigated $\mathrm{Co}_{2} \mathrm{MnGe}$ films was not specified. All values of $D$ were determined on Heusler films of typical thickness 30-80 nm using Brillouin light scattering (BLS) spectroscopy, at room temperature. The collected values of the exchange (expressed as both the spin wave stiffness $D$ and the exchange constant $A$ ), saturation magnetizations $M_{\mathrm{s}}$, and bulk Curie temperature $T_{\mathrm{C}}$ are presented in Table IV. 


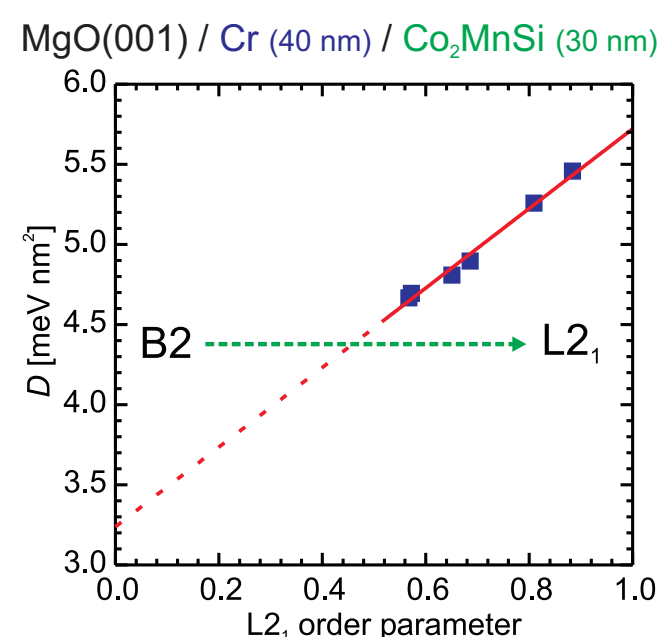

FIG. 14. Dependence of $D$ on $\mathrm{L} 2{ }_{1}$ order for $\mathrm{Co}_{2} \mathrm{MnSi}$. Data adapted from $[62,143]$. (Colour online)

Comparing the values presented in Table IV, a few qualitative trends emerge. In particular, (i) A very large change in $D$ is observed between $\mathrm{Co}_{2} \mathrm{FeSi}$ $\left(D=7.2 \mathrm{meV} \mathrm{nm}^{2}\right)$ and $\mathrm{Co}_{2} \mathrm{Cr}_{0.6} \mathrm{Fe}_{0.4} \mathrm{Al}$ or $\mathrm{Co}_{2} \mathrm{MnAl}$ (both $\sim 2.0 \mathrm{meVnm}^{2}$ ). Such a large change is associated with the introduction of only 2 valence electron per unit formula. (ii) The experimental points appear to be segregated into two branches (see Fig. 16 below), presumably related to B2- and L2 $2_{1}$-ordered compounds. In both branches, $D$ is monotonously increasing with increasing $N_{\mathrm{v}}$. The trend appears to be linear, although the investigation of further compounds would clarify the exact functional form of this dependence. (iii) The B2-ordered compounds $\mathrm{Co}_{2} \mathrm{MnAl}$ and $\mathrm{Co}_{2} \mathrm{Cr}_{0.6} \mathrm{Fe}_{0.4} \mathrm{Al}$ have similar $N_{\mathrm{v}}$ 's (28 and 27.8 valence electrons per unit formula) and very similar $D$ values.

Observations (i) - (iii) may suggest that $D$ is a function of both $N_{\mathrm{v}}$ and the crystallographic ordering. The latter observation is consistent with the above mentioned study of a series of $\mathrm{Co}_{2} \mathrm{MnSi}$ samples with a varying degree of L2 1 order [62]. The determined $D$ values are shown in Fig. 14 and linearly increase with increasing degree of $\mathrm{L} 21_{1}$ ordering.

One of the most attractive features of Heusler compounds is the possibility to tune their magnetic properties, such as $M_{\mathrm{S}}[10,39,69], T_{\mathrm{C}}[87,90]$, and Fermi level position $[60,79]$, using the chemical handle provided by $N_{\mathrm{v}}$. As was discussed above, the spin wave stiffness $D$ appears to scale with $N_{\mathrm{v}}$, for a given atomic ordering. While a quantitative description of this trend is still elusive, here we point out a few features that help to understand the dependence of $D$ on $N_{\mathrm{v}}$.

In the following, we discuss three possible contributions which may give rise to the observed dependence between the composition (and $N_{\mathrm{v}}$ ) and the Heisenberg exchange integral $J$, which is related to the spin wave stiffness $D$ (vide supra). (1) The increase of $N_{\mathrm{v}}$ adds electrons to the electronic structure. The additional elec-

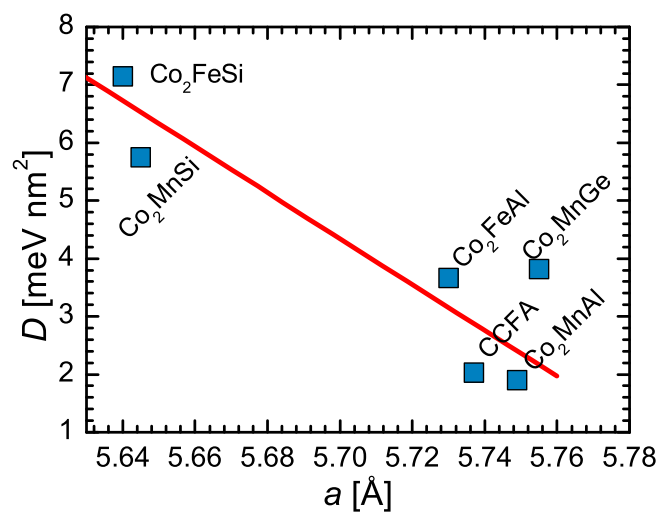

FIG. 15. Exchange stiffness $D$ as a function of the bulk lattice constant $a$. The solid line is a guide to the eye. (Colour online)

trons will be primarily found in the $t_{2 g}$ orbitals of the transition metal $M$ in the $\mathrm{Co}_{2} M^{\prime} Z$ Heusler compound [47]. Thus, increasing $N_{\mathrm{v}}$ increases the electron density that will participate in exchange. Additionally, ab-initio calculations of the element-projected density of states in $\mathrm{Co}_{2} M^{\prime} Z\left(M^{\prime}=\mathrm{Mn}, \mathrm{Fe} ; Z=\mathrm{Al}, \mathrm{Si}\right)[78,90,151]$ show that for a given non-magnetic element $Z$, substituting $M n$ by the more electronegative Fe results in a lowering of the energy of the $M^{\prime}$-based bands. This results in an improved alignment of the band energy between $M^{\prime}$ and Co atoms, providing a stronger overlap of the electronic functions, and hence a stronger exchange interaction. (2) The difference in electronegativity between Co and Si $(-0.02$ on the Pauling electronegativity scale) is much less than between $\mathrm{Co}$ and $\mathrm{Al}$ (0.27). As such, Co-Si bonding is of more covalent character than Co-Al bonding [47]. While this will impact the electronic properties, it is not clear how this affects the exchange interactions. However, this appears to be correlated to an enhanced spin wave stiffness. (3) Finally, increasing $N_{\mathrm{v}}$ is related with smaller atomic diameter of the constituent elements, leading to a contraction of the unit cell of the Heusler compounds. Hence, a stronger exchange interaction is expected due to the better overlap of orbitals, a result of the closer proximity between the magnetic elements [70, 88]. Indeed, larger spin wave stiffnesses are generally observed for smaller (bulk) lattice constants $a$ (see Fig. 15).

It is important to note that the trend between $D$ and $N_{\mathrm{v}}$ is not solely due to $N_{\mathrm{v}}$, as is the case for the magnetic moment determined by the Slater-Pauling rule [39, 69]. In particular, the same spin wave stiffness would not be expected a priori for systems having the same $N_{\mathrm{v}}$, due to generally different electronic structures, and thus exchange integrals. However, similar exchange constants and spin wave stiffnesses were observed for $\mathrm{Co}_{2} \mathrm{MnAl}$ and $\mathrm{Co}_{2} \mathrm{Cr}_{0.6} \mathrm{Fe}_{0.4} \mathrm{Al}$ (having nearly the same $N_{\mathrm{v}}=28$ and $27.8 e^{-}$/f.u., respectively).

Figure 16 compares the spin wave stiffness $D$ associated to a variety of $\mathrm{Co}_{2}$-based Heusler compounds, the 
ferromagnetic $3 d$-metals, and Fe-rich $b c c-\mathrm{Fe}_{x} \mathrm{Co}_{100-x}$ intermetallic compounds, as a function of $N_{\mathrm{v}}[10,45,62$, 80, 104, 110, 143, 149, 152-157]. As discussed above, $\mathrm{Co}_{2}$-based Heusler compounds appear to follow a roughly linear dependence of $D$ on $N_{\mathrm{v}}$, reaching a maximum value with $\mathrm{Co}_{2} \mathrm{FeSi}\left(D=7.2 \pm 0.2 \mathrm{meV} \mathrm{nm}^{2}\right)$ [147]. The $\mathrm{Fe}_{x} \mathrm{Co}_{100-x}$ compounds also provide a roughly linear dependence between $D$ and $N_{\mathrm{v}}$ in the $x$ range of $50-100$, reaching a maximum value of $D=8.0 \pm 0.5 \mathrm{meV} \mathrm{nm}^{2}$ with $\mathrm{Fe}_{53} \mathrm{Co}_{47}$ [152] which is (to the best of our knowledge), the largest spin wave stiffness ever reported. This shows that the exchange values of the $\mathrm{L} 21_{1}$-ordered $\mathrm{Co}_{2} \mathrm{MnSi}$ and $\mathrm{Co}_{2} \mathrm{FeSi}$ are extraordinarily large. They are larger than $D$ of the pure ferromagnetic $3 d$ metals, and nearly as large as the maximum value of $D$ obtained for the $\mathrm{Fe}_{x} \mathrm{Co}_{100-x}$ series. The spin wave stiffness of $\mathrm{Co}_{2} \mathrm{FeSi}$ ( $D=7.2 \mathrm{meV} \mathrm{nm}^{2}$ ), with $N_{\mathrm{v}}=30$, is to this date the highest reported for a Heusler compound [147]. This is in line with the highest $T_{\mathrm{c}}(1100 \mathrm{~K})$ and saturation magnetization $\left(6 \mu_{\mathrm{B}}\right)$ amongst the $\mathrm{Co}_{2} M^{\prime} Z$ compounds $[10,158,159]$. Not only does $\mathrm{Co}_{2} \mathrm{FeSi}$ have the highest amongst the Heusler compounds, it is only surpassed by a narrow range of $\mathrm{Co}_{100-x} \mathrm{Fe}_{x}$, where $x \approx 50$. It is also worth noting that the exchange constant $A$ of $\mathrm{Co}_{2} \mathrm{FeSi}(31.5 \mathrm{pJ} / \mathrm{m})$ is also only surpassed by $\mathrm{Fe}_{53} \mathrm{Co}_{47}$ $(38.4 \mathrm{pJ} / \mathrm{m})$, and is on par (within experimental error) with $\mathrm{Fe}_{63} \mathrm{Co}_{37}(32.1 \mathrm{pJ} / \mathrm{m})$ [152].

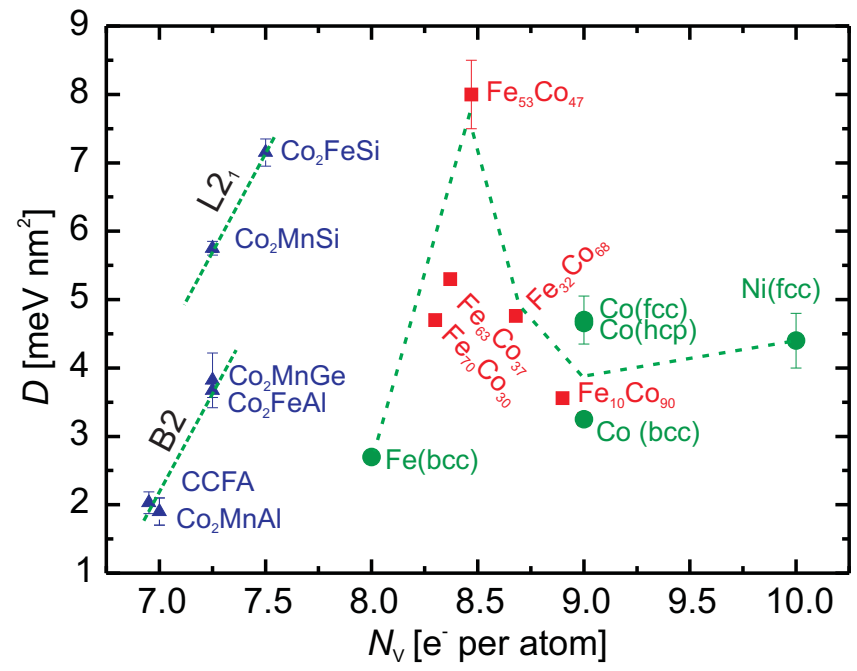

FIG. 16. Spin wave stiffness $D$ as a function of number of valence electrons $N_{\mathrm{v}}$ for $\mathrm{Co}_{2} M^{\prime} Z$ Heusler compounds, the ferromagnetic $3 d$-metals, and $b c c-\mathrm{Fe}_{x} \mathrm{Co}_{100-x}$ compounds. Lines are guides to the eye. For the Heusler compounds, multiply by 4 to obtain the number of valence electrons per unit formula. (Colour online)

It is also worth noting that higher exchange constants also correlate with higher Curie temperatures, as is shown in Table IV. This is the expected trend for Heusler compounds [90], and is in line with previously reported trends, for example the $b c c-\mathrm{Fe}_{x} \mathrm{Cr}_{1-x}$ series [160, 161].

\section{Relevance of exchange to spin polarization}

There is a known proportionality between the saturation magnetization and the spin polarization [162-165],

$$
\frac{P(T)}{P(0 K)} \propto \frac{M_{\mathrm{S}}(T)}{M_{\mathrm{S}}(0 K)}
$$

and as such it has been suggested that the spin polarization should also follow a behaviour similar to Eqn. (19) [16, 166-168], i.e.

$$
P(T)=P(0 \mathrm{~K})\left(1-\mathcal{A} T^{3 / 2}\right)
$$

where $\mathcal{A}$ is a parameter analogous to $\mathcal{B}$ in Eqn. (19). Upon inserting Eqn. (23) into Eqn. (14), one can model the temperature dependence of the TMR for a given junction. This Bloch-law-like temperature dependence has successfully been applied to the TMR temperature dependence of wide variety of TMJs [166-169], including $\mathrm{Co}_{2} \mathrm{Fe}_{0.5} \mathrm{Al}_{0.5} \mathrm{Si} / \mathrm{MgAl}_{2} \mathrm{O}_{x} / \mathrm{Co}_{50} \mathrm{Fe}_{50}$ TMJs [16]. Here we use this simple model to provide insight into how the exchange constants of various Heusler compounds will impact their spin-dependent transport properties.

It is interesting to note that for Heusler compounds, both $M_{\mathrm{s}}$ [39] and $D$ (c.f. Table IV) increase with an increasing number of valence electrons. Hence, assuming that other sources of spin depolarization are negligible, the temperature dependence should diminish (i.e. $\mathcal{B}$ is smaller) for Heusler compounds with higher $N_{\mathrm{v}}$. It was experimentally observed by Oogane and coworkers [170] that the TMR ratio of $\mathrm{Co}_{2} \mathrm{FeSi}$-based junctions decreases more slowly than that of $\mathrm{Co}_{2} \mathrm{MnSi}$-based TMJs, which is the behaviour we qualitatively expect based on the higher spin wave stiffness and saturation magnetization.

Here we have calculated the $\mathcal{B}$ values for the Heusler compounds discussed above using Eq. (20) and assuming the bulk lattice constants $a$ and saturation magnetizations $M_{\mathrm{s}}$ determined by BLS presented in Table IV. By changing the valence electron concentration by less than $10 \%, \mathcal{B}$ changes by more than an order of magnitude. Using these $\mathcal{B}$ values, $M_{\mathrm{s}}(T) / M_{\mathrm{s}}(0 \mathrm{~K})$ was calculated, as is shown in Fig. 17a. Only a very weak dependence is observed for $\mathrm{Co}_{2} \mathrm{FeSi}$ and $\mathrm{Co}_{2} \mathrm{MnSi}$, where a decrease of $\sim 1 \%$ in $M_{\mathrm{S}}$ is observed over a $350 \mathrm{~K}$ temperature change. For $\mathrm{Co}_{2} \mathrm{MnAl}$ and $\mathrm{Co}_{2} \mathrm{Cr}_{0.6} \mathrm{Fe}_{0.4} \mathrm{Al}$, this decrease is of around $12 \%$ at $350 \mathrm{~K}$.

While a good qualitative guide, it must be realized that the $\mathcal{B}$ and $\mathcal{A}$ terms of Eqns. (19) and (23) are generally not equal. For example, Shan et al. report $\mathcal{B}=4.3 \times 10^{-6} \mathrm{~K}^{-3 / 2}$ and $\mathcal{A}=3.2 \times 10^{-5} \mathrm{~K}^{-3 / 2}$ for $\mathrm{Co}_{2} \mathrm{Fe}_{0.5} \mathrm{Al}_{0.5} \mathrm{Si}$ and $\mathcal{B}=3.5 \times 10^{-6} \mathrm{~K}^{-3 / 2}$ and $\mathcal{A}=2.0 \times$ $10^{-5} \mathrm{~K}^{-3 / 2}$ for $\mathrm{Co}_{50} \mathrm{Fe}_{50}$ [16], while Shang and coworkers report $\mathcal{B}=1.23 \times 10^{-6} \mathrm{~K}^{-3 / 2}$ and $\mathcal{A} \approx 3-5 \times 10^{-5} \mathrm{~K}^{-3 / 2}$ for $\mathrm{Ni}_{80} \mathrm{Fe}_{20}$ [168]. This is mainly due to the fact that the spin polarization is a very surface dependent property, and the surface magnetization decays faster than the bulk magnetization due to surface exchange softening 


\begin{tabular}{cc}
\hline \hline Compound & $\begin{array}{c}\mathcal{B} \\
{\left[\times 10^{-6} \mathrm{~K}^{-3 / 2}\right]}\end{array}$ \\
\hline \hline $\mathrm{Co}_{2} \mathrm{FeSi}$ & 1.40 \\
$\mathrm{Co}_{2} \mathrm{MnSi}$ & 2.03 \\
$\mathrm{Co}_{50} \mathrm{Fe}_{50}[16]$ & 3.5 \\
$\mathrm{Co}_{2} \mathrm{MnGe}$ & 5.00 \\
$\mathrm{Co}_{2} \mathrm{FeAl}$ & 3.76 \\
$\mathrm{Co}_{2} \mathrm{MnAl}$ & 18.75 \\
$\mathrm{Co}_{2} \mathrm{Cr}_{0.6} \mathrm{Fe}_{0.4} \mathrm{Al}$ & 18.27 \\
\hline
\end{tabular}

TABLE V. Calculated Bloch parameters $\mathcal{B}$ for $\mathrm{Co}_{2}$-based Heusler compounds.
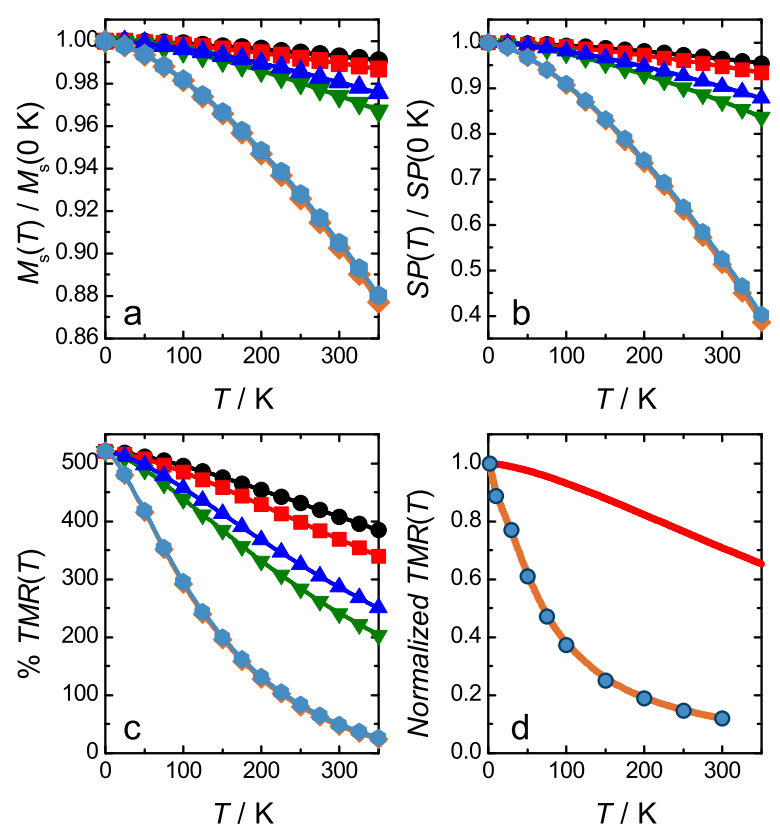

FIG. 17. Temperature dependent magnetization (a) and spin polarization (b) calculated for $\mathrm{Co}_{2} \mathrm{FeSi}(\bullet), \mathrm{Co}_{2} \mathrm{MnSi}$ $(\boldsymbol{\square}), \mathrm{Co}_{2} \mathrm{MnGe}(\boldsymbol{\nabla}), \mathrm{Co}_{2} \mathrm{FeAl}(\boldsymbol{\Delta}), \mathrm{Co}_{2} \mathrm{MnAl}(\downarrow)$, and $\mathrm{Co}_{2} \mathrm{Cr}_{0.6} \mathrm{Fe}_{0.4} \mathrm{Al}$ ( ) using Bloch $T^{3 / 2}$ power laws (see text). (c) Calculated TMR as a function of temperature for $\mathrm{Co}_{2} M^{\prime} Z / \mathrm{AlO}_{x} / \mathrm{Co}_{2} M^{\prime} Z$ TMJs (for purpose of comparison, $P(0 \mathrm{~K})$ is assumed to be 0.85 for all $\mathrm{Co}_{2} M^{\prime} Z$ ). (d) Predicted TMR ratio for $\mathrm{Co}_{2} \mathrm{MnSi} / \mathrm{AlO}_{x} / \mathrm{Co}_{2} \mathrm{MnSi}$ junction (solid line), and experimental results from Sakuraba et al. [21] for a real junction. Both data sets are normalized for comparison.

[163, 171]. The spin polarization and surface exchange also strongly depend on the surface cleanliness [172].

For the purpose of comparison without losing generality, in the following we assume $\mathcal{A} \approx 5 \mathcal{B}$. This allows to calculate $P(T) / P(0 \mathrm{~K})$, shown in Fig. 17b. The dependence naturally looks the same as for the saturation magnetization, with the major difference that the range of changes is now much larger for the Heusler compounds of interest here. As above, $\mathrm{Co}_{2} \mathrm{MnSi}$ and $\mathrm{Co}_{2} \mathrm{FeSi}$ show only a small decay of the spin polarization, whereas it is reduced by almost $60 \%$ for $\mathrm{Co}_{2} \mathrm{Cr}_{0.6} \mathrm{Fe}_{0.4} \mathrm{Al}$ and $\mathrm{Co}_{2} \mathrm{MnAl}$. Again, it is worth mentioning this drastic change is obtained by a very slight modification of the valence electron concentration.

Using the above calculated temperaturedependent spin polarizations, the TMR ratio for $\mathrm{Co}_{2} M^{\prime} Z / \mathrm{AlO}_{x} / \mathrm{Co}_{2} M^{\prime} Z$ TMJs using the compounds studied here was estimated. These are shown in Fig. 17c. Here an initial spin polarization at $0 \mathrm{~K}$ of 0.85 is assumed for all compounds. Note that a spin polarization of 0.85 would be in good agreement with reported device performance based on $\mathrm{Co}_{2} \mathrm{Fe}_{0.5} \mathrm{Al}_{0.5} \mathrm{Si}$ [16] and $\mathrm{Co}_{2} \mathrm{MnSi}$ [21] electrodes. For devices based on $\mathrm{Co}_{2} \mathrm{FeSi}$ and $\mathrm{Co}_{2} \mathrm{MnSi}$, high (>350\%) TMR ratios are seen at room temperature. Of relevance to this discussion is not the absolute TMR ratio value, but rather their decay with temperature, and the difference between various compounds.

We have compared this calculated magnetoresistance for a $\mathrm{Co}_{2} \mathrm{MnSi} / \mathrm{AlO}_{x} / \mathrm{Co}_{2} \mathrm{MnSi}$ junction to the experimental data by Sakuraba and coworkers [21] in Fig. 17d. As is clear, this simple model does not take into account all spin depolarization channels relevant to that particular TMJ device, as the TMR ratio is seen to decrease much faster than what we would anticipate if thermal magnons were the only relevant pathway to depolarization. For $\mathrm{Co}_{2} \mathrm{MnSi}$, the Fermi level lies close to the bottom of the minority spin conduction band, and the smearing of the conduction due to nonquasiparticle states could in this case dominate the spin transport properties [139]. However, we believe our estimates do provide an upper limit to what an ideal device can achieve, where tuning of the Fermi level reduces or even nullifies the effect of temperature smearing of the electronic bands, such as was demonstrated recently for $\mathrm{Co}_{2} \mathrm{Fe}_{0.5} \mathrm{Al}_{0.5} \mathrm{Si}$ [16].

Using previously published results on the effect of annealing and increased $\mathrm{L}_{1}$ ordering in $\mathrm{Co}_{2} \mathrm{MnSi}$ thin films $[62,143]$, we also evaluated the spin polarization for $\mathrm{Co}_{2} \mathrm{MnSi}$ with various degrees of $\mathrm{L}_{1}$ ordering (not shown). The reduced spin polarizations $P(300 \mathrm{~K}) / P(0 \mathrm{~K})$ changed by approximately $1.2 \%$ when comparing the range of data we had $\left(T_{\text {annealing }}\right.$ changing from $350^{\circ} \mathrm{C}$ to $500^{\circ} \mathrm{C}$ ). While the spin polarization itself, and thus the absolute TMR ratio, may increase substantially, our analysis points out that the improvement towards reducing the decay of the TMR ratio with increasing annealing temperature is most probably not due to the increase of the bulk exchange constants. On the other hand, annealing - in particular when done insitu - likely improves the interfaces (structure, cleanliness, etc.), which may improve the surface exchange and polarization [172]. While this surface exchange improvement is not an effect that would be detected in BLS experiments, it would be of the utmost importance when it comes to spin-dependent transport.

While here we have focussed on a simple model where the spin polarization decays according to a Bloch be- 
haviour, the population of thermal magnons is also an important parameter if considering spin-dependent transport when interface states near the Fermi level of the minority spin band are present. In this case, when electrons emitted by the electrode come from the minority spin band, the current is likely due to interface states [36]. Electrons excited to these interface states can tunnel through the barrier to the minority spin band interface states (when the moments of the electrodes are aligned in a parallel fashion) or the majority spin band (when in an antiparallel alignment) of the collector electrode. One channel leading to the population of the interface states is inelastic electron-magnon scattering.

Yamamoto et al. [173] have shown that when interface states are relevant, the TMR ratio (defined as $\left.\left[R_{\mathrm{AP}}-R_{\mathrm{P}}\right] / R_{\mathrm{P}}\right)$ decay with increasing temperature is largely determined by the temperature dependence of the resistance of the junction in the anti-parallel configuration $R_{\mathrm{AP}}$, whereas the resistance in the parallel state $R_{\mathrm{P}}$ is comparatively constant. This was attributed to minority-to-majority spin-flipping electron-magnon scattering populating interface states in the emitter electrode, and majority-to-minority transitions freeing up the minority interface states in the collector electrode. A higher spin wave exchange stiffness results in fewer thermally excited magnons at a given temperature. As such, electron-magnon scattering is less likely, and the tunnelling current temperature dependence in the antiparallel state, which should be absent in a true halfmetal, is (at least partially) suppressed. This would then inhibit the decay of the TMR ratio with increasing temperature. At the same time, the magnitude of the TMR ratio would be expected to increase, as $R_{\mathrm{AP}}$ would increase with respect to $R_{\mathrm{P}}$. In a qualitative sense, these considerations suggest that, as was the case for the Bloch model presented above, materials with a higher spin wave stiffness should provide TMJs whose performance are more stable with respect to temperature.

We finally present a major challenge this discussion clearly highlights towards fully understanding the temperature dependence of the magnetoresistance in Heusler-based TMJs. The strong dependence of the spin wave stiffness on composition (c.f. Table IV) suggests the decay of the TMR ratio is also a strong function of the stoichiometry of the Heusler compounds used.

For example, a two-fold difference of spin wave stiffness is found between presumably stoichiometric $\mathrm{Co}_{2} \mathrm{MnGe}(110)$ [110, 174] and non-stoichiometric $\mathrm{Co}_{2} \mathrm{Mn}_{0.77} \mathrm{Ge}_{0.42}(001)$ films [130]. It is also worth pointing to the work of Ishikawa et al. which showed that the TMR ratio can be significantly modulated when using non-stoichiometric electrodes [175]. In this study, the performance of $\mathrm{Co}_{2} \mathrm{Mn}_{x} \mathrm{Si} / \mathrm{MgO} / \mathrm{Co}_{2} \mathrm{Mn}_{x} \mathrm{Si}$ with varying amounts $x$ of $\mathrm{Mn}$ were compared to the nearlystoichiometric $\mathrm{Co}_{2} \mathrm{MnSi}(x=0.99)$ devices, for which a TMR ratio of $873 \%$ was measured $4.2 \mathrm{~K}$. For Mn-poor electrodes $(x=0.69)$, a strongly diminished TMR ratio of $\sim 200 \%$ at $4.2 \mathrm{~K}$ was reported, while this value increased to $1135 \%$ for Mn-rich $(x=1.29)$ electrodes.

These two examples demonstrate how meaningful comparisons between various devices are only possible if the stoichiometry is well characterized. Currently, much more emphasis is put on atomic ordering, which, as we discussed above, might be arguably far less important vis-à-vis the temperature dependence of TMR ratios in MTJs.

To close this section, a few guidelines towards achieving better device performance are here outlined. The comparison of our calculated TMRs with experimental results suggest there is still quite a bit of improvement possible if electronic effects (e.g. band-smearing, electron-magnon scattering, etc.) can be minimized, for example through Fermi level tuning [16]. When thermal magnon-limited, which could be a fundamental limit for the temperature dependence of the spin polarization, it is important to maximize the value of spin wave stiffness, to hinder magnon creation, thus preserving high spin polarization over a larger temperature range. Another recently proposed approach consists of doping Heusler compounds with rare earth elements, thereby suppressing the thermal excitation of magnons and preventing spin depolarization $[138,176]$.

\section{MAGNETIC DAMPING}

Since its prediction in 1996 by Berger [177] and Slonczewski [178], spin transfer torque (STT) has attracted much attention due to its potential applications in devices [13]. Figure 18 schematically explains STT. In spin transfer torque, a spin polarized current is obtained by passing current through a ferromagnet with a fixed magnetization direction. This polarized current then passes through a free-layer ferromagnet, wherein the moment is non-collinear with that of the incoming current. The spin moments of the conducting electrons align with the magnetization of the free layer, whereby transverse momentum lost by the electrons is transferred to the ferromagnet (labeled as Torque in Fig. 18). This results in a torque on the magnetization of the free layer, and the free layer's magnetization aligns with the polarization of the incoming current. Thus, current-induced magnetization reversal is achieved. For more details on STT, see the tutorial review by Ralph and Stiles [37].

For efficient spin torque transfer, one wants a high spin polarization of the incoming current, a low saturation magnetization, and low damping. During magnetization reversal, the spin transfer torque (which tries to rotate the magnetization from its equilibrium position) acts against the damping term (which tries to recover the magnetization), as is shown schematically in Fig. refScheme:STTarrows. The STT term increases with current, and reversal can only occur when the spin transfer torque overcomes the damping torque. As such, a small damping reduces the critical current density for currentinduced magnetization switching [37]. 


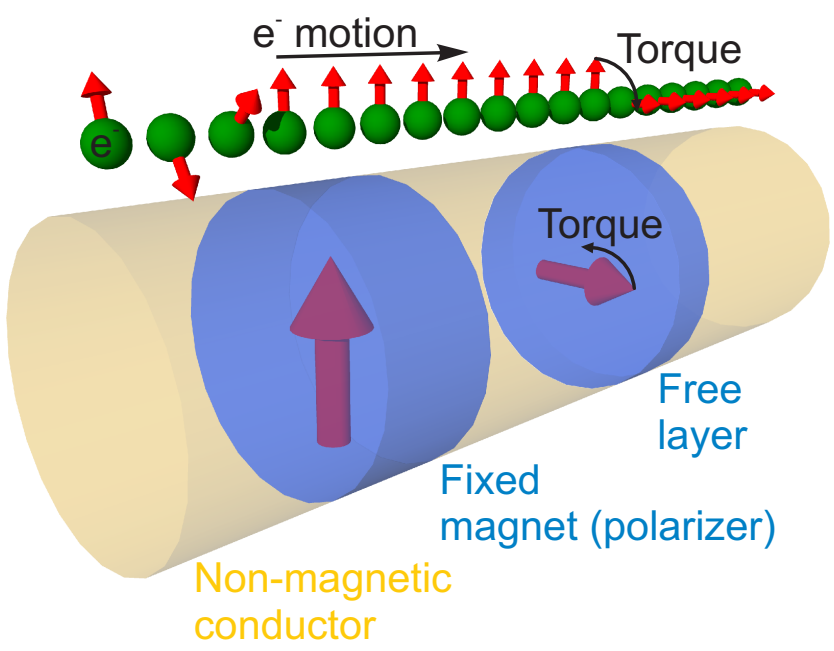

FIG. 18. Schematic representation of spin transfer torque. (Colour online)

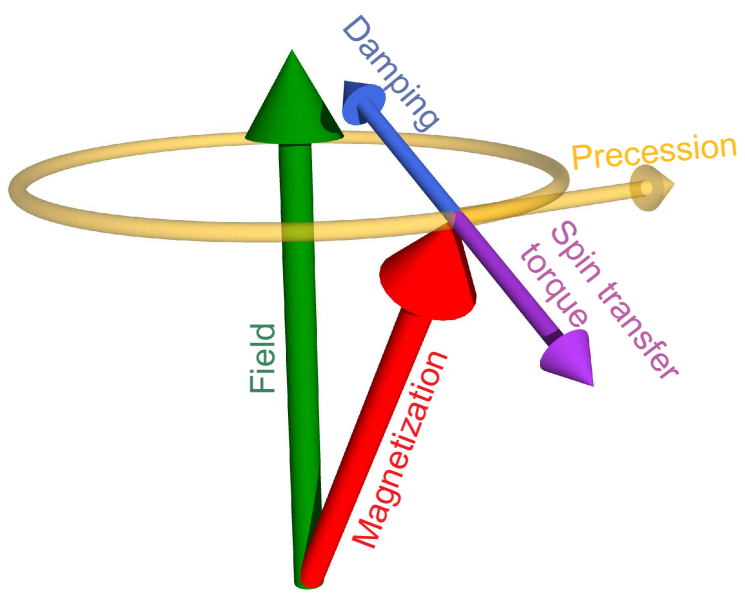

FIG. 19. Precession of the magnetization, along with damping and spin transfer torque (note that the spin transfer torque depends on the current and its direction of flow). (Colour online)

Two methods are typically used to determine the damping parameter. These two methods are ferromagnetic resonance (FMR) [179] and all-optical FMR [180], based on time-resolved MOKE. In FMR, the out-of-plane angular dependence of the linewidth of the resonance field is studied, while in all-optical FMR, the temporal evolution of the Kerr rotation in the nanosecond range is monitored, using the pump-probe method [181]. In both cases, the results are analyzed in terms of the LandauLifshitz-Gilbert (LLG) equation of motion

$$
\frac{\partial \mathbf{M}}{\partial t}=-\gamma\left(\mathbf{M} \times \mathbf{H}_{\mathrm{eff}}\right)+\frac{\alpha}{|\mathbf{M}|}\left(\mathbf{M} \times \frac{\partial \mathbf{M}}{\partial t}\right)
$$

where the first term of the right hand side describes the precession, and the second term the damping of the motion, with $\alpha$ being the Gilbert damping term (c.f. Fig. 19). Two relevant parameters are the in-

\begin{tabular}{|c|c|c|}
\hline Compound & $\alpha$ & Reference \\
\hline $\mathrm{Co}_{2} \mathrm{MnAl}$ & 0.006 & {$[184]$} \\
\hline $\mathrm{Co}_{2} \mathrm{MnAl}$ & $0.007^{1)}$ & [185] \\
\hline $\mathrm{Co}_{2} \mathrm{MnAl}$ & $0.015-0.05^{2)}$ & {$[105]$} \\
\hline $\mathrm{Co}_{2} \mathrm{MnSi}$ & $0.003 \|[100]$ & {$[106]$} \\
\hline $\mathrm{Co}_{2} \mathrm{MnSi}$ & $0.006 \|[110]$ & {$[106]$} \\
\hline $\mathrm{Co}_{2} \mathrm{MnSi}$ & $\sim 0.025^{3)}$ & {$[186]$} \\
\hline $\mathrm{Co}_{2} \mathrm{Fe}_{x} \mathrm{Mn}_{1-x}$ & .003 for $x=0.4$ & {$[187]$} \\
\hline $\mathrm{Co}_{2} \mathrm{FeSi}$ & $0.008^{4)}$ & {$[188]$} \\
\hline $\mathrm{Co}_{2} \mathrm{FeSi}$ & $0.04^{5)}$ & {$[188]$} \\
\hline $\mathrm{Co}_{2} \mathrm{FeAl}$ & $0.001^{6)}$ & {$[189]$} \\
\hline $\mathrm{Fe}$ & 0.0019 & {$[184]$} \\
\hline $\mathrm{Co}$ & 0.011 & {$[184]$} \\
\hline $\mathrm{Ni}$ & 0.064 & {$[184]$} \\
\hline $\mathrm{Ni}_{80} \mathrm{Fe}_{20}$ & 0.007 & {$[184]$} \\
\hline $\mathrm{Ni}_{80} \mathrm{Fe}_{20}$ & $0.008^{2,7)}$ & {$[190]$} \\
\hline $\mathrm{Co}_{50} \mathrm{Fe}_{50}$ & 0.0020 & {$[184]$} \\
\hline $\mathrm{Co}_{75} \mathrm{Fe}_{25}$ & 0.0041 & [184] \\
\hline $\mathrm{Co}_{80} \mathrm{Fe}_{20}$ & 0.013 & [191] \\
\hline $\mathrm{Co}_{72} \mathrm{Fe}_{18} \mathrm{~B}_{10}$ & 0.006 & [191] \\
\hline $\mathrm{Co}_{90} \mathrm{Fe}_{10}$ & 0.0043 & {$[184]$} \\
\hline \multicolumn{3}{|c|}{$\begin{array}{l}T_{\text {anneal }}=300^{\circ} \mathrm{C} ;{ }^{2)} \text { as a function of field, TR-MOKE } \\
\text { 3) polycrystalline, independent of } T_{\text {anneal }} \\
\text { 4) as-deposited (amorphous) and for } T_{\text {anneal }}=300^{\circ} \mathrm{C} \\
\left.\text { 5) } T_{\text {anneal }}=400^{\circ} \mathrm{C} ;{ }^{6}\right) T_{\text {anneal }}=600^{\circ} \mathrm{C} ;{ }^{7} \text { Small disks. }\end{array}$} \\
\hline
\end{tabular}

TABLE VI. Damping parameter for various $\mathrm{Co}_{2} M^{\prime} Z$ compounds and $3 d$ transition metals and alloys

trinsic Gilbert damping $\alpha$ and the relaxation frequency $\left(G=\alpha \gamma M_{\mathrm{s}}\right)$, also called the Landau-Lifshitz damping term [182]. The latter is used for comparison between samples, as $\alpha$ is expected to be inversely proportional to $M_{\mathrm{s}}$. The relaxation frequency is predicted to be proportional to $\xi^{2} D\left(\varepsilon_{\mathrm{F}}\right)$, where $\xi$ is the spin-orbit coupling parameter (see Eq. 6) $[93,183]$ and $D\left(\varepsilon_{\mathrm{F}}\right)$ is the density of states at the Fermi level.

Ideally, the listed desired properties can be obtained with Heusler compounds, wherein high spin polarization is expected, and the saturation magnetization is easily handled by the composition. The damping properties of $\mathrm{Co}_{2} \mathrm{MnAl}$ [105, 184, 185], $\mathrm{Co}_{2} \mathrm{MnSi}[101,106,186]$, $\mathrm{Co}_{2} \mathrm{Fe}_{x} \mathrm{Mn}_{1-x} \mathrm{Si}$ [187], $\mathrm{Co}_{2} \mathrm{FeSi}$ [188] and $\mathrm{Co}_{2} \mathrm{FeAl}$ [189] were recently studied. The obtained Gilbert damping parameters are presented in Table VI.

Also shown in Table VI are typical ferromagnetic metals and alloys [184, 190, 191]. As can be seen, the relaxation in Heusler compounds is comparable, or lower than in these materials. In particular, some Heusler compounds have lower damping than permalloy $\left(\mathrm{Ni}_{80} \mathrm{Fe}_{20}\right)$, which is one of the most widely used magnetic material.

Kubota and co-workers recently reported on the composition dependence of $\alpha$ and $G$ for the quaternary 


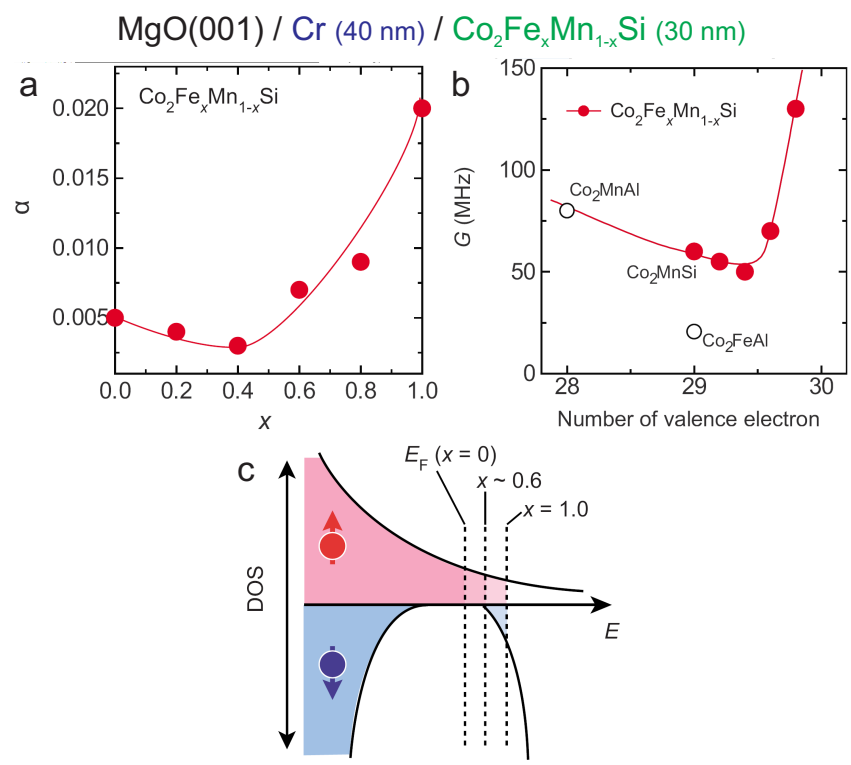

FIG. 20. Gilbert damping constants $\alpha \mathrm{Co}_{2} \mathrm{Fe}_{x} \mathrm{Mn}_{1-x} \mathrm{Si}$ Heusler thin films (a) and relaxation frequency $G\left(=\alpha \gamma M_{\mathrm{s}}\right)$ for various Heusler compounds (b). For the number of valence electron $=30\left(\mathrm{Co}_{2} \mathrm{FeSi}\right), G=416 \mathrm{MHz}$ (not plotted in the graph). (c) Expected schematic descriptions of density of states for $\mathrm{Co}_{2} \mathrm{Fe}_{x} \mathrm{Mn}_{1-x} \mathrm{Si}$. Broken lines respectively denote Fermi levels for $x=0$, about 0.6, and 1.0. Reprinted with permission from [187]. Copyright 2009, American Institute of Physics. (Colour online)

$\mathrm{Co}_{2} \mathrm{Fe}_{x} \mathrm{Mn}_{1-x} \mathrm{Si}$ compounds, by means of FMR [187]. The results are shown in Fig. 20(a-b), respectively. For both parameters, a minimum value $(\alpha=0.003)$ is seen for $\mathrm{Co}_{2} \mathrm{Fe}_{0.4} \mathrm{Mn}_{0.6} \mathrm{Si}(x=0.4)$. When considering that [183]

$$
\alpha \propto \xi^{2} D\left(\varepsilon_{\mathrm{F}}\right)
$$

small values are expected if both the spin-orbit coupling interaction $\xi$ and the density of states near the Fermi level $D\left(\varepsilon_{\mathrm{F}}\right)$ are low. The first may be expected given the low orbital moments of Heusler compounds [98]. When compared to a ferromagnetic metal, the unoccupied minority band at $\varepsilon_{\mathrm{F}}$ contributes to a lowered total density $D\left(\varepsilon_{\mathrm{F}}\right)$. The composition dependence is then interpreted as follows [187]: for $x<0.6$, a half-metallic behaviour is observed, with a low $D\left(\varepsilon_{\mathrm{F}}\right)$, resulting in low damping. For $x>0.6$ the sample would no longer be half-metallic, and the increasing density at $\varepsilon_{\mathrm{F}}$ (see Fig. 20c) results in an increasing damping. The importance of the density of states at the Fermi level was further demonstrated by Mizukami using a series of $\mathrm{Co}_{2} \mathrm{FeAl}(001)$ grown on $\mathrm{MgO}$ and annealed at various temperatures [189]. The annealing increased the B2 ordering parameter ( $S$ in Fig. 21), and the relaxation frequency $G$, determined by FMR, decreases with improved B2 order. Also shown in this Figure are $a b$ initio calculations where the atomic disorder is implemented within the coherent potential approximation [38]. The two trends show congruous behaviour, such that the order-damping relationship is, at

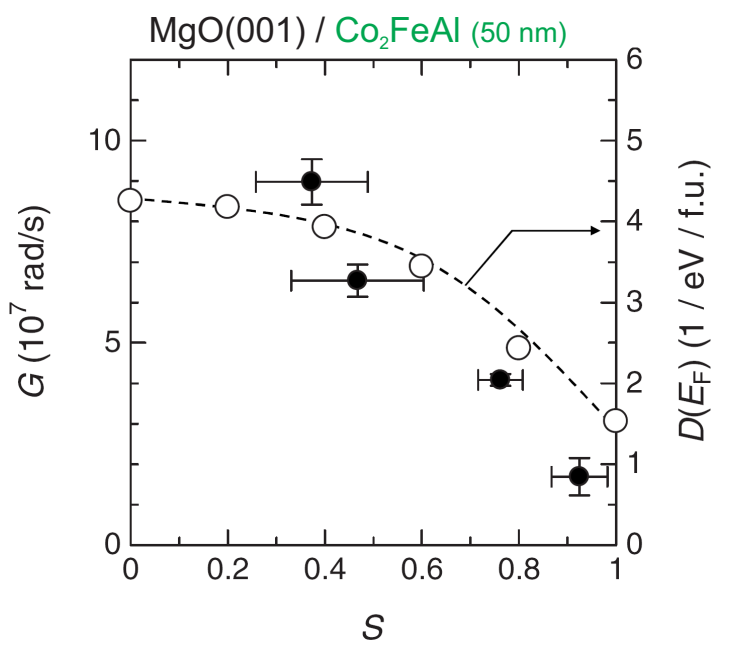

FIG. 21. Relaxation frequency $G(\bullet)$ and the calculated total DOS at Fermi level $D\left(\varepsilon_{\mathrm{F}}\right)$ (o) as a function of long range order parameter $S$ for B2 order. Reprinted with permission from [189]. Copyright 2009, American Institute of Physics. (Colour online)

least qualitatively, understood in terms of the vanishing density of states at the Fermi level. It is worth mentioning that for $T_{\text {anneal }}=600^{\circ}$ leading to $S \approx 0.92, \alpha=0.001$ and $G=2 \times 10^{7} \mathrm{rad} / \mathrm{s}$, which compares very favorably to the value of $(3.5 \pm 5) \times 10^{7} \mathrm{rad} / \mathrm{s}$ found for $\mathrm{Fe}_{0.73} \mathrm{~V}_{0.27}$, the lowest of all $3 d$ ferromagnetic alloys [192].

As a final example, the relaxation properties of $\mathrm{Co}_{2} \mathrm{MnAl}$ were investigated by all-optical FMR [105]. In this case, a comprehensive study of the relaxation at various frequencies was achieved by varying the applied magnetic field. In that study, it was also demonstrated that the precession results from an ultrafast modification of the magnetocrystalline anisotropy field.

The study of relaxation in $\mathrm{Co}_{2} M^{\prime} Z$ Heusler compounds is still in its initial stages. The first example of STT magnetization switching in Heuslerbased spin-valve nanopillars was recently reported by Jukegawa and coworkers [193]. The critical current density for magnetization switching in the studied $\mathrm{Co}_{2} \mathrm{Fe}_{0.5} \mathrm{Al}_{0.5} \mathrm{Si} / \mathrm{MgO} / \mathrm{Co}_{2} \mathrm{Fe}_{0.5} \mathrm{Al}_{0.5} \mathrm{Si}$ nanopillars was $9.3 \times 10^{6} \mathrm{~A} / \mathrm{cm}^{2}$. As anticipated, this current is lower than the critical current densities encountered in permalloy $\left(\sim 1.1 \times 10^{7} \mathrm{~A} / \mathrm{cm}^{2}[194]\right)$ and $\mathrm{Co} / \mathrm{Cu} / \mathrm{Co}$ based devices (where current densities in the $2-12 \times 10^{7} \mathrm{~A} / \mathrm{cm}^{2}$ range were reported $[195,196])$. As such, we can anticipate the advantageous characteristics of Heusler compounds will be harnessed, and more investigations are expected as Heusler compounds are integrated into other STT-based devices, such as domain-wall nanowire shift register (also known as racetrack) memory [197] or STT magnetic random access memory (STT-MRAM) [13]. 


\section{CONCLUSIONS}

We have provided an overview of the magnetic properties of cobalt-based full-Heusler compounds. The complexity of these systems, which arises from the wide range of chemical compositions, atomic structures, and defects, and the impact these have on their electronic properties, makes them particularly challenging, and interesting to study. While ab initio calculations have been a guide towards the development of materials with advantageous properties, the experimental investigation of their magnetic properties remains the ultimate test of our understanding of these materials. While advances are continuously being made, further work will be necessary before all the complex interactions between chemical and physical characteristics, and their impact on the magnetic anisotropy, exchange, and damping are fully understood. Crucial to this task is a thorough control of ordering, stoichiometry, and interface engineering.

It is however clear that both theory and experiment have, over the past recent years, shown beyond a doubt that Heusler compounds are indeed in many respects very attractive towards novel applications in emerging spintronic devices. Whether this is due to their high spin polarization, tunable properties, or low magnetic damping, it appears evident that even though Heusler compounds are more than a century old, they are still in their infancy, and still have much fascinating physics to reveal.

\section{ACKNOWLEDGEMENTS}

ST is grateful to the Alexander von Humboldt foundation for a postdoctoral fellowship during his stay in TU Kaiserslautern, and thanks G. Wolf (TU Kaiserslautern) for a critical reading of this manuscript and stimulating discussions. We thank H.J. Elmers, G. Fecher, C. Felser, G. Jakob, and M. Jourdan, from Johannes-Gutenberg U. (Mainz), Y. Ando, T. Kubota, M. Oogane, and Y. Sakuraba (Tohoku U.), M. Yamamoto (Hokkaido U.), K. Inomata (NIMS), J. Kübler (Darmstadt Technical U.), R. Schäfer (IFW Dresden), K. Postava (Technical U. Ostrava), and M. Aeschlimann and M. Cincetti (TU Kaiserslautern), and members of their respective groups, for useful discussions and collaborations during our investigations of $\mathrm{Co}_{2} M^{\prime} Z$ compounds. Financial support by the Deutsche Forschungsgemeinschaft Research Unit 559, "New Materials with High Spin Polarization" is gratefully acknowledged.
[1] F. Heusler, Verh. Dtsch. Phys. Ges. 5, 219 (1903).

[2] H. H. Potter, Proc. Phys. Soc. 41, 135 (1929).

[3] A. J. Bradley and J. W. Rodgers, Proc. R. Soc. London A 144, 340 (1934).

[4] G. B. Johnston and E. O. Hall, J. Phys. Chem. Solids 29, 193 (1968).

[5] P. J. Webster, Contemp. Phys. 10, 559 (1969).

[6] Y. Ishikawa and Y. Noda, AIP Conf. Proc. 24, 145 (1975).

[7] D. C. Price, J. Phys. F: Metal Phys. 8, 933 (1978).

[8] M. B. Stearns, J. Appl. Phys. 50, 2060 (1979).

[9] P. G. Van Engen, K. H. J. Buschow, R. Jongebreur, and M. Erman, Appl. Phys. Lett. 42, 202 (1983).

[10] S. Wurmehl, G. H. Fecher, H. C. Kandpal, V. Ksenofontov, C. Felser, H.-J. Lin, and J. Morais, Phys. Rev. B 72, 184434 (2005).

[11] R. A. de Groot, F. M. Mueller, P. G. van Engen, and K. H. J. Buschow, Phys. Rev. Lett. 50, 2024 (1983).

[12] J. M. D. Coey, M. Venkatesan, and M. A. Bari, in High Magnetic Fields, Vol. 595 of Lecture Notes in Physics, edited by C. Berthier, L. P. Lévy, and G. Martinez (Springer, Berlin, 2002), pp. 377-396.

[13] C. Chappert, A. Fert, and F. Nguyen Van Dau, Nature Mater. 6, 813 (2007).

[14] C. Felser, G. Fecher, and B. Balke, Angew. Chem. Int. Ed. 46, 668 (2007).

[15] K. Inomata, N. Ikeda, N. Tezuka, R. Goto, S. Sugimoto, M. Wojcik, and E. Jedryka, Sci. Technol. Adv. Mater. 9, 014101 (2008).

[16] R. Shan, H. Sukegawa, W. H. Wang, M. Kodzuka, T. Furubayashi, T. Ohkubo, S. Mitani, K. Inomata, and K. Hono, Phys. Rev. Lett. 102, 246601 (2009).
[17] T. Taira, T. Ishikawa, N. Itabashi, K.-i. Matsuda, T. Uemura, and M. Yamamoto, J. Phys. D: Appl. Phys. 42, 084015 (2009).

[18] T. Tsunegi, Y. Sakuraba, M. Oogane, K. Takanashi, and Y. Ando, Appl. Phys. Lett. 93, 112506 (2008).

[19] T. Ishikawa, S. Hakamata, K.-i. Matsuda, T. Uemura, and M. Yamamoto, J. Appl. Phys. 103, 07 A919 (2008).

[20] N. Tezuka, N. Ikeda, S. Sugimoto, and K. Inomata, Jpn. J. Appl. Phys. 46, L454 (2007).

[21] Y. Sakuraba, M. Hattori, M. Oogane, Y. Ando, H. Kato, A. Sakuma, T. Miyazaki, and H. Kubota, Appl. Phys. Lett. 88, 192508 (2006).

[22] N. Tezuka, N. Ikeda, S. Sugimoto, and K. Inomata, Appl. Phys. Lett. 89, 252508 (2006).

[23] P. A. Dowben and R. Skomski, J. Appl. Phys. 95, 7453 (2004).

[24] P. M. Tedrow and R. Meservey, Phys. Rev. B 7, 318 (1973).

[25] R. J. Soulen Jr., J. M. Byers, M. S. Osofsky, B. Nadgorny, T. Ambrose, S. F. Cheng, P. R. Broussard, C. T. Tanaka, J. Nowak, J. S. Moodera, A. Barry, and J. M. D. Coey, Science 282, 85 (1998).

[26] I. Galanakis, J. Phys. Condens. Matter 14, 6329 (2002).

[27] S. J. Hashemifar, P. Kratzer, and M. Scheffler, Phys. Rev. Lett. 94, 096402 (2005).

[28] S. Ishida, T. Masaki, S. Fujii, and S. Asano, Physica B 245, 1 (1998)

[29] K. Nagao, Y. Miura, and M. Shirai, Phys. Rev. B 73, 104447 (2006).

[30] Y. Miura, H. Uchida, Y. Oba, K. Nagao, and M. Shirai, J. Phys. Condens. Matter 19, 365228 (2007). 
[31] Y. Miura, H. Uchida, Y. Oba, K. Abe, and M. Shirai, Phys. Rev. B 78, 064416 (2008).

[32] S. Picozzi and A. J. Freeman, J. Phys.: Condens. Matter 19, 315215 (2007).

[33] S. Picozzi, A. Continenza, and A. J. Freeman, Phys. Rev. B 69, 094423 (2004).

[34] M. Kallmayer, H. Schneider, G. Jakob, H. J. Elmers, K. Kroth, H. Kandpal, U. Stumm, and C. Cramm, Appl. Phys. Lett. 88, 072506 (2006).

[35] M. Kallmayer, H. Schneider, G. Jakob, H. J. Elmers, B. Balke, and C. Cramm, J. Phys. D: Appl. Phys. 40, 1552 (2007).

[36] P. Mavropoulos, M. Ležaić, and S. Blügel, Phys. Rev. B 72, 174428 (2005).

[37] D. C. Ralph and M. D. Stiles, J. Magn. Magn. Mater. 320, 1190 (2008).

[38] J. Kübler, Theory of Itinerant Electron Magnetism, revised ed. (Oxford University Press, New York, 2009).

[39] I. Galanakis, P. Mavropoulos, and P. H. Dederichs, J. Phys. D: Appl. Phys. 39, 765 (2006).

[40] I. Galanakis and P. H. Dederichs, in Half-metallic Alloys - Fundamentals and Applications, Vol. 676 of Lecture Notes in Physics, edited by I. Galanakis and P. Dederichs (Springer, Berlin, 2005), pp. 1-39.

[41] G. E. Bacon and J. S. Plant, J. Phys. F: Metal Phys. 1, 524 (1971).

[42] P. Eckerlin and H. Kandler, Alloys and Compounds of $d$-Elements with Main Group Elements Part 2 (LandoltBörnstein-Group III Condensed Matter vol 6) (Springer, Berlin, 1985), p. 233.

[43] P. J. Webster and K. R. A. Ziebeck, Alloys and Compounds of d-Elements with Main Group Elements Part 2 (Landolt-Börnstein-Group III Condensed Matter vol 19C) (Springer, Berlin, 1988), pp. 75-185.

[44] H. J. Elmers, S. Wurmehl, G. H. Fecher, G. Jakob, C. Felser, and G. Schönhense, Appl. Phys. A 79, 557 (2004).

[45] P. J. Webster, J. Phys. Chem. Solids 32, 1221 (1971).

[46] R. Y. Umetsu, K. Kobayashi, A. Fujita, K. Oikawa, R. Kainuma, K. Ishida, N. Endo, K. Fukamichi, and A. Sakuma, Phys. Rev. B 72, 214412 (2005).

[47] H. C. Kandpal, G. H. Fecher, and C. Felser, J. Phys. D: Appl. Phys. 40, 1507 (2007).

[48] Y. Takamura, R. Nakane, and S. Sugahara, J. Appl. Phys. 105, 07B109 (2009).

[49] B. Balke, S. Wurmehl, G. H. Fecher, C. Felser, M. C. M. Alves, F. Bernardi, and J. Morais, Appl. Phys. Lett. 90, 172501 (2007).

[50] T. M. Nakatani, A. Rajanikanth, Z. Gercsi, Y. K. Takahashi, K. Inomata, and K. Hono, J. Appl. Phys. 102, 033916 (2007).

[51] B. Ravel, M. P. Raphael, V. G. Harris, and Q. Huang, Phys. Rev. B 65, 184431 (2002).

[52] B. Ravel, J. O. Cross, M. P. Raphael, V. G. Harris, R. Ramesh, and V. Saraf, Appl. Phys. Lett. 81, 2812 (2002).

[53] B. A. Collins, Y. Zhong, Y. S. Chu, L. He, and F. Tsui, J. Vac. Sci. Technol. B 25, 999 (2007).

[54] L. Basit, C. Wang, C. A. Jenkins, B. Balke, V. Ksenofontov, G. H. Fecher, C. Felser, E. Mugnaioli, U. Kolb, S. A. Nepijko, G. Schonhense, and M. Klimenkov, J. Phys. D: Appl. Phys. 42, 084018 (2009).

[55] S. Wurmehl and J. T. Kohlhepp, J. Phys. D: Appl. Phys. 41, $173002(2008)$.
[56] K. Inomata, M. Wojcik, E. Jedryka, N. Ikeda, and N. Tezuka, Phys. Rev. B 77, 214425 (2008).

[57] S. Wurmehl, J. T. Kohlhepp, H. J. M. Swagten, B. Koopmans, M. Wojcik, B. Balke, C. G. F. Blum, V. Ksenofontov, G. H. Fecher, and C. Felser, Appl. Phys. Lett. 91, 052506 (2007).

[58] S. Wurmehl, J. T. Kohlhepp, H. J. M. Swagten, B. Koopmans, C. G. F. Blum, V. Ksenofontov, H. Schneider, G. Jakob, and G. Reiss, J. Phys. D: Appl. Phys. 42, 084017 (2009).

[59] K. Kobayashi, R. Umetsu, A. Fujita, K. Oikawa, R. Kainuma, K. Fukamichi, and K. Ishida, J. Alloys Compd. 399, 60 (2005).

[60] B. Balke, G. H. Fecher, H. C. Kandpal, C. Felser, K. Kobayashi, E. Ikenaga, J.-J. Kim, and S. Ueda, Phys. Rev. B 74, 104405 (2006).

[61] K. Kobayashi, R. Umetsu, K. Ishikawa, R. Kainuma, and K. Ishida, J. Magn. Magn. Mater. 310, 1794 (2007).

[62] O. Gaier, J. Hamrle, S. J. Hermsdoerfer, H. Schultheiß B. Hillebrands, Y. Sakuraba, M. Oogane, and Y. Ando, J. Appl. Phys. 103, 103910 (2008).

[63] W. Wang, H. Sukegawa, R. Shan, T. Furubayashi, and K. Inomata, Appl. Phys. Lett. 92, 221912 (2008).

[64] Y. Miura, K. Nagao, and M. Shirai, Phys. Rev. B 69, 144413 (2004).

[65] C. Palmstrøm, MRS Bulletin 10, 725 (2003).

[66] J. Mathon and A. Umerski, Phys. Rev. B 63, 220403 (2001).

[67] W. H. Butler, X.-G. Zhang, T. C. Schulthess, and J. M. MacLaren, Phys. Rev. B 63, 054416 (2001).

[68] S. Picozzi, A. Continenza, and A. J. Freeman, Phys. Rev. B 66, 094421 (2002).

[69] I. Galanakis, P. H. Dederichs, and N. Papanikolaou, Phys. Rev. B 66, 174429 (2002).

[70] E. Şaşioğlu, L. M. Sandratskii, P. Bruno, and I. Galanakis, Phys. Rev. B 72, 184415 (2005).

[71] Z. Gercsi and K. Hono, J. Phys. Condens. Matter 19, 326216 (2007).

[72] S. Fujii, S. Sugimura, S. Ishida, and S. Asano, J. Phys. Condens. Matter 2, 8583 (1990).

[73] S. Ishida, S. Fujii, S. Kashiwagi, and S. Asano, J. Phys. Soc. Jpn. 64, 2152 (1995).

[74] J. Kübler, A. R. Williams, and C. B. Sommers, Phys. Rev. B 28, 1745 (1983).

[75] M. Oogane, Y. Sakuraba, J. Nakata, H. Kubota, Y. Ando, A. Sakuma, and T. Miyazaki, J. Phys. D: Appl. Phys. 39, 834 (2006).

[76] B. Balke, G. H. Fecher, and C. Felser, Appl. Phys. Lett. 90, 242503 (2007).

[77] I. Galanakis, J. Phys. Condens. Matter 16, 3089 (2004).

[78] K. Özdoğan, E. Şaşioğlu, B. Aktaş, and I. Galanakis, Phys. Rev. B 74, 172412 (2006).

[79] G. H. Fecher and C. Felser, J. Phys. D: Appl. Phys. 40, 1582 (2007).

[80] T. Block, C. Felser, G. Jakob, J. Ensling, B. Mühling, P. Gütlich, and R. J. Cava, J. Solid State Chem. 176, 646 (2003).

[81] K. H. J. Buschow, P. G. van Engen, and R. Jongebreur, J. Magn. Magn. Mater. 38, 1 (1983).

[82] P. J. Brown, K. U. Neumann, P. J. Webster, and K. R. A. Ziebeck, J. Phys. Condens. Matter 12, 1827 (2000).

[83] J. Kübler, Physica B 127, 257 (1984). 
[84] J. C. Slater, J. Appl. Phys. 8, 385 (1937).

[85] L. Pauling, Phys. Rev. 54, 899 (1938).

[86] R. M. Bozorth, Phys. Rev. 79, 887 (1950).

[87] G. H. Fecher, H. C. Kandpal, S. Wurmehl, C. Felser, and G. Schönhense, J. Appl. Phys. 99, 08J106 (2006).

[88] Y. Kurtulus, R. Dronskowski, G. D. Samolyuk, and V. P. Antropov, Phys. Rev. B 71, 014425 (2005).

[89] J. Thoene, S. Chadov, G. Fecher, C. Felser, and J. Kübler, J. Phys. D: Appl. Phys. 42, 084013 (2009).

[90] J. Kübler, G. H. Fecher, and C. Felser, Phys. Rev. B 76, 024414 (2007).

[91] J. Kübler, J. Phys. Condens. Matter 18, 9795 (2006).

[92] A. Hubert and R. Schäfer, Magnetic Domains - The Analysis of Magnetic Microstructures (Springer, Berlin, 1998).

[93] J. Stöhr and H. C. Siegmann, Magnetism, From Fundamentals to Nanoscale Dynamics, Vol. 152 of Solid-State Sciences (Springer Verlag, Berlin Heidelberg, 2006).

[94] H. J. Elmers, S. Wurmehl, G. H. Fecher, G. Jakob, C. Felser, and G. Schönhense, J. Magn. Magn. Mater. 272, 758 (2004).

[95] S. Wurmehl, G. H. Fecher, K. Kroth, F. Kronast, H. A. Dürr, Y. Takeda, Y. Saitoh, K. Kobayashi, H.-J. Lin, G. Schönhense, and C. Felser, J. Phys. D: Appl. Phys. 39, 803 (2006).

[96] K. Miyamoto, A. Kimura, K. Iori, K. Sakamoto, T. Xie, T. Moko, S. Qiao, M. Taniguchi, and K. Tsuchiya, J. Phys. Condens. Matter 16, S5797 (2004).

[97] N. D. Telling, P. S. Keatley, G. van der Laan, R. J. Hicken, E. Arenholz, Y. Sakuraba, M. Oogane, Y. Ando, K. Takanashi, A. Sakuma, and T. Miyazaki, Phys. Rev. B 78, 184438 (2008).

[98] I. Galanakis, Phys. Rev. B 71, 012413 (2005).

[99] M. Sargolzaei, M. Richter, K. Koepernik, I. Opahle, H. Eschrig, and I. Chaplygin, Phys. Rev. B 74, 224410 (2006).

[100] B. Hillebrands, in Modern Techniques for Characterizing Magnetic Materials, edited by Y. Zhu (Springer, Berlin Heidelberg, 2005), pp. 543-578.

[101] R. Yilgin and B. Aktas, in Advances in Nanoscale Magnetism, Vol. 122 of Springer Proceedings in Physics, edited by B. Aktas and F. Mikailov (Springer, Berlin Heidelberg, 2008), pp. 37-65.

[102] T. Uemura, T. Yano, K.-i. Matsuda, and M. Yamamoto, Thin Solid Films 515, 8013 (2007).

[103] T. Uemura, T. Yano, K.-i. Matsuda, and M. Yamamoto, J. Magn. Magn. Mater. 310, e696 (2007).

[104] O. Gaier, J. Hamrle, S. Trudel, A. C. Parra, B. Hillebrands, C. H. E. Arbelo, and M. Jourdan, J. Phys. D: Appl. Phys. 42, 084004 (2009).

[105] Y. Liu, L. R. Shelford, V. V. Kruglyak, R. J. Hicken, Y. Sakuraba, M. Oogane, Y. Ando, and T. Miyazaki, J. Appl. Phys. 101, 09C106 (2007).

[106] R. Yilgin, Y. Sakuraba, M. Oogane, S. Mizumaki, Y. Ando, and T. Miyazaki, Jpn. J. Appl. Phys. 46, L205 (2007).

[107] F. Y. Yang, C. H. Shang, C. L. Chien, T. Ambrose, J. J. Krebs, G. A. Prinz, V. I. Nikitenko, V. S. Gornakov, A. J. Shapiro, and R. D. Shull, Phys. Rev. B 65, 174410 (2002).

[108] T. Ambrose, J. J. Krebs, and G. A. Prinz, Appl. Phys. Lett. 76, 3280 (2000).

[109] T. Ambrose, J. J. Krebs, and G. A. Prinz, J. Appl. Phys. 87, 5463 (2000).
[110] M. Belmeguenai, F. Zighem, Y. Roussigné, S.-M. Chérif, P. Moch, K. Westerholt, G. Woltersdorf, and G. Bayreuther, Phys. Rev. B 79, 024419 (2009).

[111] P. K. Muduli, W. C. Rice, L. He, and F. Tsui, J. Magn. Magn. Mater. 320, L141 (2008).

[112] P. K. Muduli, W. C. Rice, L. He, B. A. Collins, Y. S. Chu, and F. Tsui, J. Phys. Condens. Matter 21, 296005 (2009).

[113] M. Hashimoto, J. Herfort, H.-P. Schönherr, and K. H. Ploog, J. Appl. Phys. 98, 104902 (2005).

[114] M. Hashimoto, J. Herfort, H.-P. Schönherr, and K. H. Ploog, Appl. Phys. Lett. 87, 102506 (2005).

[115] J. Hamrle, S. Blomeier, O. Gaier, B. Hillebrands, R. Schäfer, and M. Jourdan, J. Appl. Phys. 100, 103904 (2006).

[116] W. H. Wang, X. B. Ren, G. H. Wu, M. Przybylski, J. Barthel, and J. Kirschner, IEEE Trans. Magn. 41, 2805 (2005).

[117] W. H. Wang, M. Przybylski, W. Kuch, L. I. Chelaru, J. Wang, Y. F. Lu, J. Barthel, H. L. Meyerheim, and J. Kirschner, Phys. Rev. B 71, 144416 (2005).

[118] T. Uemura, Y. Imai, S. Kawagishi, K.-i. Matsuda, and M. Yamamoto, Physica E 40, 2025 (2008).

[119] M. J. Pechan, C. Yu, D. Carr, and C. J. Palmstrøm, J. Magn. Magn. Mater. 286, 340 (2005).

[120] A. Hirohata, H. Kurebayashi, S. Okamura, N. Tezuka, and K. Inomata, IEEE Trans. Magn. 41, 2802 (2005).

[121] M. R. Paudel, C. S. Wolfe, H. M. A. Patton, J. Simonson, I. Dubenko, N. Ali, and S. Stadler, J. Appl. Phys. 105, 07E902 (2009).

[122] J. J. Krebs, B. T. Jonker, and G. A. Prinz, J. Appl. Phys. 61, 2596 (1987).

[123] L. J. Singh, Z. H. Barber, A. Kohn, A. K. Petford-Long, Y. Miyoshi, Y. Bugoslavsky, and L. F. Cohen, J. Appl. Phys. 99, 013904 (2006).

[124] Š. Višňovský, Czech. J. Phys. B 36, 1424 (1986).

[125] K. Postava, D. Hrabovský, J. Pištora, A. R. Fert, Š. Višnovský, and T. Yamaguchi, J. Appl. Phys. 91, 7293 (2002).

[126] For $\varphi=0^{\circ}$ ( $\varphi$ is the angle of light incidence) $\mathcal{A}=$ $\left(\tilde{n}_{\mathrm{o}}^{2} \varphi\right) /\left[\tilde{n}^{2}\left(\tilde{n}_{\mathrm{o}}^{2}-\tilde{n}^{2}\right)\right]$ and $\mathcal{B}=\tilde{n}_{\mathrm{o}} /\left[\tilde{n}\left(\tilde{n}_{\mathrm{o}}^{2}-\tilde{n}^{2}\right)\right]\left(\tilde{n}, \tilde{n}_{\mathrm{o}}\right.$ are the complex refractive indices of the material and incident medium, respectively). This neglects any overlying layer (capping layer, barrier), and the finite thickness of the magneto-optically active film.

[127] J. Hamrle, S. Blomeier, O. Gaier, B. Hillebrands, K. Postava, H. Schneider, G. Jakob, and C. Felser, J. Phys. D: Appl. Phys. 40, 1563 (2007).

[128] J. Hamrle, S. Blomeier, O. Gaier, B. Reuscher, A. Brodyanski, M. Kopnarski, K. Postava, H. Schneider, G. Jacob, C. Felser, and B. Hillebrands, J. Phys. D: Appl. Phys. 40, 1558 (2007).

[129] S. Trudel, G. Wolf, H. Schultheiß, J. Hamrle, B. Hillebrands, T. Kubota, and Y. Ando, Rev. Sci. Instrum. 81, 026105 (2010).

[130] S. Trudel, J. Hamrle, B. Hillebrands, T. Taira, and M. Yamamoto, J. Appl. Phys. 107, (2010), in press, doi:10.1063/1.3296350.

[131] Q.-M. Zhong, A. S. Arrott, B. Heinrich, and Z. Celinski, J. Appl. Phys. 67, 4448 (1990).

[132] Q.-M. Zhong, A. S. Arrott, B. Heinrich, and Z. Celinski, J. Magn. Magn. Mater 104-107, 1837 (1992).

[133] K. Postava, H. Jaffres, A. Schuhl, F. Nguyen Van Dau, M. Goiran, and A. R. Fert, J. Magn. Magn. Mater. 172, 
199 (1997).

[134] S.-s. Yan, R. Schreiber, P. Grünberg, and R. Schäfer, J. Magn. Magn. Mater. 210, 309 (2000).

[135] M. Buchmeier, R. Schreiber, D. E. Bürgler, and C. M. Schneider, Phys. Rev. B 79, 064402 (2009).

[136] R. M. Osgood III, S. D. Bader, B. M. Clemens, R. L. White, and H. Matsuyama, J. Magn. Magn. Mater. 182, 297 (1998).

[137] M. Jullière, Phys. Lett. A 54, 225 (1975).

[138] A. Rajanikanth, Y. K. Takahashi, and K. Hono, J. Appl. Phys. 105, 063916 (2009), and references therein.

[139] L. Chioncel, Y. Sakuraba, E. Arrigoni, M. I. Katsnelson, M. Oogane, T. Miyazaki, E. Burzo, and A. I. Lichtenstein, Phys. Rev. Lett. 100, 086402 (2008).

[140] V. Drewello, J. Schmalhorst, A. Thomas, and G. Reiss, Phys. Rev. B 77, 014440 (2008).

[141] M. Pajda, J. Kudrnovský, I. Turek, V. Drchal, and P. Bruno, Phys. Rev. B 64, 174402 (2001).

[142] C. Kittel, Introduction to Solid State Physics, 7th ed. (Wiley, New York, 1996).

[143] J. Hamrle, O. Gaier, S.-G. Min, B. Hillebrands, Y. Sakuraba, and Y. Ando, J. Phys. D: Appl. Phys. 42, 084005 (2009).

[144] L. Ritchie, G. Xiao, Y. Ji, T. Y. Chen, C. L. Chien, M. Zhang, J. Chen, Z. Liu, G. Wu, and X. X. Zhang, Phys. Rev. B 68, 104430 (2003).

[145] A. Aharoni, Introduction to the Theory of Ferromagnetism, 2nd ed. (Oxford University Press, Oxford, 2000).

[146] B. Hillebrands, Phys. Rev. B 41, 530 (1990).

[147] O. Gaier, J. Hamrle, S. Trudel, B. Hillebrands, H. Schneider, and G. Jakob, J. Phys. D: Appl. Phys. 42, 232001 (2009).

[148] G. Fecher, 2008, private communication.

[149] T. Kubota, J. Hamrle, Y. Sakuraba, O. Gaier, M. Oogane, A. Sakuma, B. Hillebrands, K. Takanashi, and Y. Ando, J. Appl. Phys. 106, 113907 (2009).

[150] N. Tezuka, N. Ikeda, A. Miyazaki, S. Sugimoto, M. Kikuchi, and K. Inomata, Appl. Phys. Lett. 89, 112514 (2006).

[151] K. Özdoğan, B. Aktaş, I. Galanakis, and E. Şaşioğlu, J. Appl. Phys. 101, 073910 (2007).

[152] X. Liu, R. Sooryakumar, C. J. Gutierrez, and G. A. Prinz, J. Appl. Phys. 75, 7021 (1994).

[153] X. Liu, M. M. Steiner, R. Sooryakumar, G. A. Prinz, R. F. C. Farrow, and G. Harp, Phys. Rev. B 53, 12166 (1996).

[154] T. Fukuda, M. Yuge, T. Terai, and T. Kakeshita, J. Phys.: Conf. Series 51, 307 (2006).

[155] S. M. Rezende, M. A. Lucena, A. Azevedo, F. M. de Aguiar, J. R. Fermin, and S. S. P. Parkin, J. Appl. Phys. 93, 7717 (2003).

[156] G. Shirane, V. J. Minkiewicz, and R. Nathans, J. Appl. Phys. 39, 383 (1968).

[157] S. Shallcross, A. E. Kissavos, V. Meded, and A. V. Ruban, Phys. Rev. B 72, 104437 (2005).

[158] S. Wurmehl, G. H. Fecher, H. C. Kandpal, V. Ksenofontov, C. Felser, and H. J. Lin, Appl. Phys. Lett. 88, 032503 (2006).

[159] S. Wurmehl, G. H. Fecher, V. Ksenofontov, F. Casper, U. Stumm, C. Felser, H.-J. Lin, and Y. Hwu, J. Appl. Phys. 99, 08J103 (2006).

[160] A. Katsuki, Brit. J. Appl. Phys. 18, 199 (1967).
[161] E. Wohlfarth, Quantum Theory of the Solid State (Academic Press, New York, 1966), p. 485.

[162] M. B. Stearns, J. Magn. Magn. Mater. 5, 167 (1977).

[163] D. T. Pierce, R. J. Celotta, J. Unguris, and H. C. Siegmann, Phys. Rev. B 26, 2566 (1982).

[164] D. Mauri, D. Scholl, H. C. Siegmann, and E. Kay, Phys. Rev. Lett. 61, 758 (1988).

[165] R. Meservey and P. M. Tedrow, Phys. Rep. 238, 173 (1994).

[166] J. S. Moodera, J. Nowak, and R. J. M. van de Veerdonk, Phys. Rev. Lett. 80, 2941 (1998).

[167] S. G. Wang, R. C. C. Ward, G. X. Du, X. F. Han, C. Wang, and A. Kohn, Phys. Rev. B 78, 180411 (2008).

[168] C. H. Shang, J. Nowak, R. Jansen, and J. S. Moodera, Phys. Rev. B 58, R2917 (1998).

[169] T. Hagler, R. Kinder, and G. Bayreuther, J. Appl. Phys. 89, 7570 (2001).

[170] M. Oogane, M. Shinano, Y. Sakuraba, and Y. Ando, J. Appl. Phys. 105, 07C903 (2009).

[171] J. Mathon and S. B. Ahmad, Phys. Rev. B 37, 660 (1988).

[172] D. Mauri, D. Scholl, H. C. Siegmann, and E. Kay, Phys. Rev. Lett. 61, 758 (1988).

[173] T. Ishikawa, N. Itabashi, T. Taira, K.-i. Matsuda, T. Uemura, and M. Yamamoto, Appl. Phys. Lett. 94, 092503 (2009).

[174] M. Belmeguenai, F. Zighem, G. Woltersdorf, Y. Roussigné, S.-M. Chérif, K. Westerholt, and G. Bayreuther, J. Magn. Magn. Mater. 321, 750 (2009).

[175] T. Ishikawa, H.-x. Liu, T. Taira, K.-i. Matsuda, and M. Yamamoto, Appl. Phys. Lett. 95, 232512 (2009).

[176] E. Burzo, I. Balazs, L. Chioncel, E. Arrigoni, and F. Beiuseanu, Phys. Rev. B 80, 214422 (2009).

[177] L. Berger, Phys. Rev. B 54, 9353 (1996).

[178] J. C. Slonczewski, J. Magn. Magn. Mater. 159, L1 (1996).

[179] B. Heinrich, in Ultrahin Magnetic Structures, edited by B. Heinrich and J. A. C. Bland (Springer, New York, 1994), Vol. 2, Chap. 3, pp. 195-222.

[180] M. van Kampen, C. Jozsa, J. T. Kohlhepp, P. LeClair, L. Lagae, W. J. M. de Jonge, and B. Koopmans, Phys. Rev. Lett. 88, 227201 (2002).

[181] B.-C. Choi and M. Freeman, in Modern Techniques for Characterizing Magnetic Materials, edited by Y. Zhu (Springer, Berlin Heidelberg, 2005), pp. 517-542.

[182] B. Heinrich, in Ultrahin Magnetic Structures, edited by J. A. C. Bland and B. Heinrich (Springer, New York, 2005), Vol. 3, Chap. 5, pp. 143-210.

[183] V. Kamberský, Can. J. Phys. 48, 2906 (1970).

[184] M. Oogane, T. Wakitani, S. Yakata, R. Yilgin, Y. Ando, A. Sakuma, and T. Miyazaki, Jpn. J. Appl. Phys. 45, 3889 (2006).

[185] R. Yilgin, M. Oogane, S. Yakata, Y. Ando, and T. Miyazaki, IEEE Trans. Magn. 41, 2799 (2005).

[186] R. Yilgin, M. Oogane, Y. Ando, and T. Miyazaki, J. Magn. Magn. Mater. 310, 2322 (2007).

[187] T. Kubota, S. Tsunegi, M. Oogane, S. Mizukami, T. Miyazaki, H. Naganuma, and Y. Ando, Appl. Phys. Lett. 94, 122504 (2009).

[188] M. Oogane, R. Yilgin, M. Shinano, S. Yakata, Y. Sakuraba, Y. Ando, and T. Miyazaki, J. Appl. Phys. 101, 09J501 (2007).

[189] S. Mizukami, D. Watanabe, M. Oogane, Y. Ando, Y. Miura, M. Shirai, and T. Miyazaki, J. Appl. Phys. 105, 
07D306 (2009).

[190] W. K. Hiebert, A. Stankiewicz, and M. R. Freeman, Phys. Rev. Lett. 79, 1134 (1997).

[191] C. Bilzer, T. Devolder, J.-V. Kim, G. Counil, C. Chappert, S. Cardoso, and P. P. Freitas, J. Appl. Phys. 100, 053903 (2006).

[192] C. Scheck, L. Cheng, I. Barsukov, Z. Frait, and W. E. Bailey, Phys. Rev. Lett. 98, 117601 (2007).

[193] H. Sukegawa, S. Kasai, T. Furubayashi, S. Mitani, and K. Inomata, Appl. Phys. Lett. 96, 042508 (2010).

[194] P. M. Braganca, I. N. Krivorotov, O. Ozatay, A. G. F. Garcia, N. C. Emley, J. C. Sankey, D. C. Ralph, and
R. A. Buhrman, Appl. Phys. Lett. 87, 112507 (2005).

[195] T. Yang, A. Hirohata, T. Kimura, and Y. Otani, J. Appl. Phys. 99, 073708 (2006).

[196] Y. Otani, H. Kubota, A. Fukushima, H. Maehara, S. Yuasa, and Y. Suzuki, J.Magn. Magn. Mater. 310, 2026 (2007).

[197] S. S. P. Parkin, M. Hayashi, and L. Thomas, Science 320, 190 (2008). 